Research Article

\title{
Quadratic Cost Minimax Optimal Control Problems for a Semilinear Viscoelastic Equation with Long Memory
}

\author{
Jin-soo Hwang (iD \\ Department of Mathematics Education, College of Education, Daegu University, Jillyang, Gyeongsan, Gyeongbuk, Republic \\ of Korea
}

Correspondence should be addressed to Jin-soo Hwang; jshwang@daegu.ac.kr

Received 25 December 2020; Revised 5 May 2021; Accepted 11 May 2021; Published 24 May 2021

Academic Editor: Dimitri Mugnai

Copyright (c) 2021 Jin-soo Hwang. This is an open access article distributed under the Creative Commons Attribution License, which permits unrestricted use, distribution, and reproduction in any medium, provided the original work is properly cited.

In this paper, we study the quadratic cost minimax optimal control problems for a semilinear viscoelastic equation with long memory. A global well-posedness theorem regarding the solutions to its Cauchy problem is given. We formulate the minimax control problem with bilinear control inputs and corresponding disturbances. Under some assumptions, we prove the existence of optimal pairs and give necessary optimality conditions for optimal pairs in some observation cases.

\section{Introduction}

We study the minimax optimal control problems for the following viscoelastic equation with long memory:

$$
\frac{\partial^{2} y}{\partial t^{2}}-\Delta y-\int_{0}^{t} k(t-s) \Delta y(s) \mathrm{d} s+|y|^{\gamma} y=u y+f .
$$

As is widely known, equation (1) is considered a typical model of viscoelastic waves with a long-memory damping. By analyzing the above equation with various boundary conditions or additional linear or nonlinear terms, one can study the eventual properties of the viscoelastic dynamics, such as exponential decay or blowup. We can find many research articles in this direction, to name just a few, refer to Cavalcanti et al. [1, 2], Xiao and Liang [3], and Messaoudi [4] and references therein. However, the research on other applications including optimal control or identification problems for the above state equation is few.

In this paper, we study the quadratic cost bilinear minimax control problems for equation (1) with the control or disturbance function $u$. For the bilinear control problems, we refer to Bradley and Lenhart [5-7] in which they studied the bilinear control problems on linear hyperbolic state equations. Recently, Belmiloudi [8] employed the bilinear minimax control framework to study robust control in a linear parabolic state equation. In [9], we extended the results in [8] to a quasilinear beam equation by proving the Fréchet differentiability of the nonlinear solution map.

The minimax control methods have been employed by many mathematicians for various control problems (see Arada and Raymond [10], Lasiecka and Triggiani [11], and Li and Yong [12]). As explained in [8,9], in this paper, the minimax control framework is employed to consider the effects of disturbance (or noise) in control inputs such that a cost function achieves its optimum (minimum) even in the case of the worst disturbances of the system. For the purpose, we replace the bilinear multiplier $u$ in equation (1) by $c+\eta$, where $c$ is a control variable that belongs to the admissible control set $\mathcal{U}_{\text {ad }}$ and $\eta$ is a disturbance (or noise) that belongs to the admissible disturbance set $\mathscr{V}_{\text {ad }}$. We also introduce the following cost function to be minimized within $\mathscr{U}_{\mathrm{ad}}$ and maximized within $\mathscr{V}_{\text {ad }}$ :

$$
\mathscr{J}(c, \eta)=\frac{1}{2}\left\|\mathscr{C} y(c+\eta)-Y_{d}\right\|_{\mathscr{M}}^{2}+\frac{\alpha}{2}\|c\|_{L^{2}(0, T)}^{2}-\frac{\beta}{2}\|\eta\|_{L^{2}(0, T)}^{2},
$$

where $y(c+\eta)$ is the solution of equation (1) with $u=c+\eta$, $\mathscr{M}$ is a Hilbert space of observation variables, $\mathscr{C}$ is a continuous observation operator, $Y_{d} \in \mathscr{M}$ is a desired value, and 
the positive constants $\alpha$ and $\beta$ are the relative weights of the second and third terms on the right-hand side of (2).

In this paper, our goal is to find and characterize the optimal controls of the cost function (2) even in the worst disturbances through the control input in equation (1). This leads to the problem of proving the existence of an admissible control $c^{*} \in \mathscr{U}_{\mathrm{ad}}$ and disturbance (or noise) $\eta^{*} \in \mathscr{V}_{\text {ad }}$ such that $\left(c^{*}, \eta^{*}\right)$ is a saddle point of the functional $\mathscr{J}(c, \eta)$ of $(2)$, that is,

$$
\mathscr{J}\left(c^{*}, \eta\right) \leq \mathscr{J}\left(c^{*}, \eta^{*}\right) \leq \mathscr{J}\left(c, \eta^{*}\right), \quad \forall(c, \eta) \in \mathscr{U}_{\mathrm{ad}} \times \mathscr{V}_{\mathrm{ad}} .
$$

Then, we derive optimality conditions for such $\left(c^{*}, \eta^{*}\right)$ in (3) corresponding to some observation cases. As in [9], we use the term optimal pair to indicate such a saddle point $\left(c^{*}, \eta^{*}\right)$ in $(3)$.

As a main tool to prove the existence of an optimal pair $\left(c^{*}, \eta^{*}\right)$ satisfying (3), we use the minimax theorem in infinite dimensions given in Barbu and Precupanu [13]. For this, we prove the Fréchet differentiability of the nonlinear solution map and its local Lipschitz continuity.

Next, we derive the necessary optimal conditions for some observation cases that should be satisfied by the optimal pairs in these observation cases. To derive these conditions, we refer to the previous results in [5-7] dealing with linear problems. Especially, we deduce the necessary optimality condition for the velocity distribution observation case which is physically meaningful. We propose an appropriate adjoint system for the velocity observation case. To author's knowledge, this is a newly developed adjoint system to deal with the second-order Volterra-type state equation. Finally, when deducing the optimality condition for the velocity observation case, we use the regularization method proposed by Lions [14] (cf. [15]) to overcome some difficulties. This is another novelty of the paper.

\section{Preliminaries}

2.1. Notations and Assumptions. Let $\Omega$ be an open, connected, and bounded set of $R^{n}(n \leq 3)$ with the smooth boundary $\Gamma$. We set $Q=(0, T) \times \Omega$ and $\Sigma=(0, T) \times \Gamma$ for $T>0$. We consider the following viscoelastic equation with long memory with the Dirichlet boundary condition:

$$
\left\{\begin{array}{l}
\frac{\partial^{2} y}{\partial t^{2}}-\Delta y-\int_{0}^{t} k(t-s) \Delta y(s) \mathrm{d} s+|y|^{\gamma} y=u y+f, \quad \text { in } Q \\
y=0, \quad \text { on } \Sigma \\
y(0, x)=y_{0}(x), \\
\frac{\partial y}{\partial t}(0, x)=y_{1}(x), \quad \text { in } \Omega
\end{array}\right.
$$

where $y_{0}(x)$ and $y_{1}(x)$ are given initial values, $f$ is a given forcing function, $k$ is a memory kernel function, and $u$ is a bilinear forcing control function applied to the system together with displacement $y$.

We denote $\|\cdot\|_{p}=\|\cdot\|_{L^{p}(\Omega)}, H=L^{2}(\Omega)$, and $V=H_{0}^{1}$ $(\Omega)$, where $p \geq 1$. We endow the space $V$ with the usual scalar product and norm:

$$
\begin{aligned}
((\phi, \psi))_{V} & =(\nabla \phi, \nabla \psi)_{2}, \\
\|\phi\|_{V} & =\|\nabla \phi\|_{2}, \quad \forall \phi, \psi \in V,
\end{aligned}
$$

where $(\cdot, \cdot)_{2}$ means the inner product on $H$. Let us denote the topological dual space of $V$ by $V^{\prime}\left(=H^{-1}(\Omega)\right)$ and the dual pairing between $V$ and $V^{\prime}$ by $\langle\cdot, \cdot\rangle_{V, V^{\prime}}$. Then, operator $A$ defined by

$$
\langle A \phi, \psi\rangle_{V^{\prime}, V}=((\phi, \psi))_{V}, \quad \forall \phi, \psi \in V,
$$

is a nonnegative self-adjoint operator with domain $D(A)=H^{2}(\Omega) \cap V$. Also, it is obvious that each natural topological imbedding

$$
D(A) \hookrightarrow V \hookrightarrow H \hookrightarrow V^{\prime}
$$

is continuous and compact.

According to Adams [16], we know the following embeddings:

$$
\begin{gathered}
H^{1}(\Omega) \hookrightarrow L^{p}(\Omega), \quad \forall p<\infty \text { if } n=1,2, p=6 \text { if } n=3, \\
H^{1}(\Omega) \hookrightarrow C(\bar{\Omega}) \quad \text { if } n=1, \\
H^{2}(\Omega) \hookrightarrow C(\bar{\Omega}) \quad \text { if } n=2,3 .
\end{gathered}
$$

Throughout this paper, the following assumptions will be effective:

$\left(H_{1}\right)$ For the exponent $\gamma$ in equation (4), we assume that

$$
\begin{array}{cl}
0<\gamma<\infty & \text { if } n=1,2, \\
0<\gamma \leq 2 & \text { if } n=3 .
\end{array}
$$

For the above $\gamma$, we define the nonlinear function $g: \mathbf{R} \longrightarrow \mathbf{R}$ by $g(s)=|s|^{\gamma} s$. Then, it is easily verified that $g \in C^{1}(\mathbf{R})$ and $g^{\prime}(s)=(\gamma+1)|s|^{\gamma}$. For given $\phi \in$ $V$, we can deduce from (8) and (11) that the nonlinear operator $g(\cdot): V \longrightarrow H$, defined by $g(\phi)(x)=g(\phi$ $(x))$, is well defined.

$\left(H_{2}\right)$ For the memory kernel function $k(\cdot)$ in equation (4), we assume that

$$
k \in C^{1}([0, T])
$$

Now, equation (4) can be rewritten as the abstract initial value problem described by the following second-order semilinear Volterra integrodifferential system:

$$
\left\{\begin{array}{l}
y^{\prime \prime}+A y+\int_{0}^{t} k(t-s) A y \mathrm{~d} s+g(y)=u y+f, \quad \text { in }(0, T), \\
y(0)=y_{0}, \quad y^{\prime}(0)=y_{1} .
\end{array}\right.
$$


2.2. Well-Posedness and Continuity of the Solution Map. The Hilbert space $\mathscr{W}(0, T)$ of the weak solutions of equation (13) is defined by

$$
\begin{aligned}
\mathscr{W}(0, T)= & \left\{\phi \mid \phi \in L^{2}(0, T ; V), \phi^{\prime} \in L^{2}(0, T ; H), \phi^{\prime \prime}\right. \\
& \left.\in L^{2}\left(0, T ; V^{\prime}\right)\right\},
\end{aligned}
$$

equipped with the norm

$$
\|\phi\|_{\mathscr{W}(0, T)}=\left(\|\phi\|_{L^{2}(0, T ; V)}^{2}+\left\|\phi^{\prime}\right\|_{L^{2}(0, T ; H)}^{2}+\left\|\phi^{\prime \prime}\right\|_{L^{2}\left(0, T ; V^{\prime}\right)}^{2}\right)^{1 / 2},
$$

where $\phi^{\prime}$ and $\phi^{\prime \prime}$ denote the first- and second-order time derivatives of $\phi$ in the sense of distribution.

Definition 1. A function $y$ is said to be a weak solution of equation (13) if $y \in \mathscr{W}(0, T)$ and $y$ satisfies

$$
\begin{cases}\langle y \prime \prime(\cdot), \varphi\rangle_{V^{\prime}, V}+\left(\left(y(\cdot)+k^{*} y(\cdot), \varphi\right)\right)_{V}+(g(y(\cdot)), \varphi)_{2}=(u(\cdot) y(\cdot)+f(\cdot), \varphi)_{2}, & \text { for all } \varphi \in V \text { in the sense of } \mathscr{D}^{\prime}(0, T) \\ y(0)=y_{0}, & y^{\prime}(0)=y_{1},\end{cases}
$$

where $\mathscr{D}^{\prime}(0, T)$ is the space of distributions in $(0, T)$.

Remark 1. For the existence and uniqueness of weak or strong solutions of equation (13) without the integral term, one can refer to the results of an abstract semilinear wave equation given in Teman ([17], pp. 212-214). In addition, by referring to the results in [18] dealing with the Volterra-type semilinear evolution equations, one can verify the wellposedness of equation (13).

Let $X$ be a Banach space. Set

$C_{w}([0, T] ; X)=\left\{f \in L^{\infty}(0, T ; X) \mid\langle f(\cdot), \xi\rangle_{X, X^{\prime}} \in C([0, T]), \forall \xi \in X^{\prime}\right\}$.

Lemma 1. Assume that

$$
\left(y_{0}, y_{1}, u, f\right) \in V \times H \times L^{2}(0, T) \times L^{2}(0, T ; H)
$$

and $y$ is a corresponding weak solution of equation (13). Then, we can verify that

$$
\begin{gathered}
y \in C_{w}([0, T] ; V), \\
y^{\prime} \in C_{w}([0, T] ; H) .
\end{gathered}
$$

Proof. By regarding $-g(y)+u y+f$ in equation (13) as $f\left(t, y, y^{\prime}\right)$ in [18] and noting (18), we can use the result of [18] to obtain

$$
y \in \mathscr{W}(0, T) \cap L^{\infty}(0, T ; V) \cap W^{1, \infty}(0, T ; H) .
$$

Then, as shown in [18] (cf. Lions and Magenes [19], pp. 275-278), we can obtain (19) from (20). This completes the proof.

The following lemma is used frequently and importantly in this paper.
Lemma 2. Let $y$ be a weak solution of equation (13) with condition (18). Then, for each $t \in[0, T]$, we have the following energy equality:

$$
\begin{aligned}
& \left\|y^{\prime}(t)\right\|_{2}^{2}+\|y(t)\|_{V}^{2}+\frac{2}{\gamma+2}\|y(t)\|_{\gamma+2}^{\gamma+2}+2\left(\left(k^{*} y(t), y(t)\right)\right)_{V} \\
& =2 \int_{0}^{t}\left(\left(k^{\prime} * y, y\right)\right)_{V} \mathrm{~d} s+2 \int_{0}^{t} k(0)\|y\|_{V}^{2} \mathrm{~d} s \\
& \quad+2 \int_{0}^{t}\left(u y, y^{\prime}\right)_{2} \mathrm{~d} s+2 \int_{0}^{t}\left(f, y^{\prime}\right)_{2} \mathrm{~d} s \\
& +\left\|y_{1}\right\|_{2}^{2}+\left\|y_{0}\right\|_{V}^{2}+\frac{2}{\gamma+2}\left\|y_{0}\right\|_{\gamma+2}^{\gamma+2} .
\end{aligned}
$$

Proof. By (19) and the uniform boundedness theorem, $y(t) \in V$ and $y^{\prime}(t) \in H$ for each $t \in[0, T]$. Thus, we know that all terms in (21) are meaningful. From (18) and (20), we know that $-g(y)+u y+f \in L^{2}(0, T ; H)$. Thus, by applying the result in Proposition 2.1 of [18] to equation (13), we obtain

$$
\begin{aligned}
& \left\|y^{\prime}(t)\right\|_{2}^{2}+\|y(t)\|_{V}^{2}+2((k * y(t), y(t)))_{V} \\
& =2 \int_{0}^{t}\left(\left(k^{\prime} * y, y\right)\right)_{V} \mathrm{~d} s+2 \int_{0}^{t} k(0)\|y\|_{V}^{2} \mathrm{~d} s \\
& \quad-2 \int_{0}^{t}\left(g(y), y^{\prime}\right)_{2} \mathrm{~d} s+2 \int_{0}^{t}\left(u y, y^{\prime}\right)_{2} \mathrm{~d} s+2 \int_{0}^{t}\left(f, y^{\prime}\right)_{2} \mathrm{~d} s \\
& \quad+\left\|y_{1}\right\|_{2}^{2}+\left\|y_{0}\right\|_{V^{\prime}}^{2}
\end{aligned}
$$

Since

$$
\left.\int_{0}^{t}(g(y)) y^{\prime}\right)_{2} \mathrm{~d} s=\frac{1}{(\gamma+2)} \frac{\mathrm{d}}{\mathrm{d} t}\|y(t)\|_{\gamma+2}^{\gamma+2},
$$


we can combine (22) and (23) to obtain (21).

This completes the proof.

With the help of (21) or (22), we can address the following theorem.

Throughout this paper, we use $C$ as a generic constant and omit the integral variables in any definite integrals without confusion.

Theorem 1. For given condition (18), let $y$ be the corresponding weak solution of equation (13). Then, we can assure that $y \in C([0, T] ; V) \cap C^{1}([0, T] ; H)$. Moreover, the solution mapping $p=\left(y_{0}, y_{1}, f, u\right) \longrightarrow y(p)$ of $\mathscr{P} \equiv V \times H$ $\times L^{2}(0, T ; H) \times L^{2}(0, T) \quad$ into $\quad \mathcal{S}(0, T) \equiv \mathscr{W} \quad(0, T) \cap C($ $[0, T] ; V) \cap C^{1}([0, T] ; H)$ is locally Lipschitz continuous. Indeed, for each $p_{1}=\left(y_{0}^{1}, y_{1}^{1}, f_{1}, u_{1}\right) \in \mathscr{P}$ and $p_{2}=$ $\left(y_{0}^{2}, y_{1}^{2}, f_{2}, u_{2}\right) \in \mathscr{P}$, the following is satisfied:

$\left\|y\left(p_{1}\right)-y\left(p_{2}\right)\right\|_{\mathcal{S}(0, T)}$

$\leq C\left(\left\|y_{0}^{1}-y_{0}^{2}\right\|_{V}^{2}+\left\|y_{1}^{1}-y_{1}^{2}\right\|_{2}^{2}+\left\|f_{1}-f_{2}\right\|_{L^{2}(0, T ; H)}^{2}+\left\|u_{1}-u_{2}\right\|_{L^{2}(0, T)}^{2}\right)^{1 / 2}$

$\equiv C\left\|p_{1}-p_{2}\right\|_{\mathscr{P}}$ where $C>0$ is a constant depending on the data.

Proof. Referring to Dautray and Lions ([20], pp. 578-581), we can make use of $(22)$ to show that $t \longrightarrow\left\|y^{\prime}(t)\right\|_{2}^{2}+$ $\|y(t)\|_{V}^{2}$ is continuous, which implies the following:

$$
y \in C([0, T] ; V) \cap C^{1}([0, T] ; H) .
$$

Thus, every weak solution $y$ of equation (13) with data condition (18) exists in $\mathcal{S}(0, T)$. From this result, we show inequality (24): we denote $y_{1}-y_{2} \equiv y\left(p_{1}\right)-y\left(p_{2}\right)$ by $\phi$. Then, from equation (13), we can know that $\phi$ satisfies the following in the weak sense:

$$
\left\{\begin{array}{l}
\phi^{\prime \prime}+A \phi+\int_{0}^{t} k(t-s) A \phi \mathrm{d} s+(\gamma+1) B\left(y_{1}, y_{2}\right) \phi=u_{1} \phi+\left(u_{1}-u_{2}\right) y_{2}+f_{1}-f_{2}, \quad \text { in }(0, T) \\
\phi(0)=y_{0}^{1}-y_{0}^{2} \\
\phi^{\prime}(0)=y_{1}^{1}-y_{1}^{2}
\end{array}\right.
$$

where

$$
B\left(y_{1}, y_{2}\right)=\int_{0}^{1}\left|\theta y_{1}+(1-\theta) y_{2}\right|^{\gamma} \mathrm{d} \theta \text {. }
$$

To obtain the estimation for $\phi$ in equation (13), we apply energy equality (22) to equation (26). Then, the energy equality for $\phi$ can be given by

By using $y_{i} \in \mathcal{S}(0, T)(i=1,2),(8),(11)$, and the Hölder inequality, we note that

$$
\begin{aligned}
\left\|(\gamma+1) B\left(y_{1}, y_{2}\right) \phi\right\|_{2} & \leq C\left\|\left(\left|y_{1}\right|+\left|y_{2}\right|\right)^{\gamma} \phi\right\|_{2} \\
& \leq C\left(\left\|y_{1}\right\|_{V}+\left\|y_{2}\right\|_{V}\right)^{\gamma}\|\phi\|_{V} \\
& \leq C\left(\left\|y_{1}\right\|_{C([0, T] ; V)}+\left\|y_{2}\right\|_{C([0, T] ; V)}\right)^{\gamma}\|\phi\|_{V} \\
& \leq C\left(\left\|y_{1}\right\|_{\mathcal{S}(0, T)}+\left\|y_{2}\right\|_{\mathcal{S}(0, T)}\right)^{\gamma}\|\phi\|_{V} .
\end{aligned}
$$

$$
\begin{aligned}
& \left\|\phi^{\prime}(t)\right\|_{2}^{2}+\|\phi(t)\|_{V}^{2} \\
& =-2((k * \phi(t), \phi(t)))_{V}+2 \int_{0}^{t}\left(\left(k^{\prime} * \phi, \phi\right)\right)_{V} \mathrm{~d} s+2 \int_{0}^{t} k(0)\|\phi\|_{V}^{2} \mathrm{~d} s \\
& \quad-2(\gamma+1) \int_{0}^{t}\left(B\left(y_{1}, y_{2}\right) \phi, \phi^{\prime}\right)_{2} \mathrm{~d} s+2 \int_{0}^{t}\left(u_{1} \phi+\left(u_{1}-u_{2}\right) y_{2}, \phi^{\prime}\right)_{2} \mathrm{~d} s \\
& \quad+2 \int_{0}^{t}\left(f_{1}-f_{2}, \phi^{\prime}\right)_{2} \mathrm{~d} s+\left\|\phi_{1}\right\|_{2}^{2}+\left\|\phi_{0}\right\|_{V}^{2} .
\end{aligned}
$$


By (28), we can verify that

$$
\begin{aligned}
& \left|2(\gamma+1) \int_{0}^{t}\left(B\left(y_{1}, y_{2}\right) \phi, \phi^{\prime}\right)_{2} \mathrm{~d} s\right| \\
& \leq C\left(\left\|y_{1}\right\|_{\mathcal{S}(0, T)}+\left\|y_{2}\right\|_{\mathcal{S}(0, T)}\right)^{\gamma} \int_{0}^{t}\|\phi\|_{V}\left\|\phi^{\prime}\right\|_{2} \mathrm{~d} s \\
& \leq C \int_{0}^{t}\left(\|\phi\|_{V}^{2}+\left\|\phi^{\prime}\right\|_{2}^{2}\right) \mathrm{d} s .
\end{aligned}
$$

By estimating other terms in the right-hand side of (29) as in [18], we can get

$$
\begin{aligned}
\left\|\phi^{\prime}(t)\right\|_{2}^{2}+\|\phi(t)\|_{V}^{2} \leq & C\left(\int_{0}^{t}\left(\left|u_{1}\right|^{2}+1\right)\left(\left\|\phi^{\prime}\right\|_{2}^{2}+\|\phi\|_{V}^{2}\right) \mathrm{d} s+\left\|\phi^{\prime}(0)\right\|_{2}^{2}\right. \\
& \left.+\|\phi(0)\|_{V}^{2}+\left\|f_{1}-f_{2}\right\|_{L^{2}(0, T ; H)}^{2}+\left\|\left(u_{1}-u_{2}\right) y_{2}\right\|_{L^{2}(0, T ; H)}^{2}\right) .
\end{aligned}
$$

Here, we note that

$$
\begin{aligned}
\left\|\left(u_{1}-u_{2}\right) y_{2}\right\|_{L^{2}(0, T ; H)}^{2} & \leq\left\|u_{1}-u_{2}\right\|_{L^{2}(0, T)}^{2}\left\|y_{2}\right\|_{C([0, T] ; H)}^{2} \\
& \leq C\left\|y_{2}\right\|_{\mathcal{S}(0, T)}^{2}\left\|u_{1}-u_{2}\right\|_{L^{2}(0, T)}^{2} .
\end{aligned}
$$

Thus, plugging (32) to (31) and applying Gronwall's lemma, we have the following estimation:

$$
\begin{aligned}
\left\|\phi^{\prime}(t)\right\|_{2}^{2}+\|\phi(t)\|_{V}^{2} \leq & C \exp \left(C\left(\left\|u_{1}\right\|_{L^{2}(0, T)}^{2}+1\right)\right)\left(\left\|\phi^{\prime}(0)\right\|_{2}^{2}+\|\phi(0)\|_{V}^{2}\right. \\
& \left.+\left\|f_{1}-f_{2}\right\|_{L^{2}(0, T ; H)}^{2}+\left\|u_{1}-u_{2}\right\|_{L^{2}(0, T)}^{2}\right) \\
& \equiv C\left\|p_{1}-p_{2}\right\|_{\mathscr{P}}^{2},
\end{aligned}
$$

which immediately implies

$$
\|\phi\|_{C([0, T] ; V) \cap C^{1}([0, T] ; H)} \leq C\left\|p_{1}-p_{2}\right\|_{\mathscr{P}} .
$$

Since $A \in \mathscr{L}\left(V, V^{\prime}\right)$ is an isomorphism, by conducting similar estimations in equation (26), we can obtain from (34) that

$$
\left\|\phi^{\prime \prime}\right\|_{L^{2}\left(0, T ; V^{\prime}\right)} \leq C\left\|p_{1}-p_{2}\right\|_{\mathscr{P}}
$$

Hence, by (34) and (35), we can prove (24).

This completes the proof.

Remark 2. From Theorem 1, for fixed $\left(y_{0}, y_{1}, f\right) \in$ $V \times H \times L^{2}(0, T ; H)$, we can define the continuous solution map from the term $u \in L^{2}(0, T)$ to $y(p) \in \mathcal{S}(0, T)$ satisfying equation (13). Indeed, for each $p_{i}=\left(y_{0}, y_{1}, f, u_{i}\right) \in \mathscr{P}(i=1,2)$, we have

$$
\left\|y\left(p_{1}\right)-y\left(p_{2}\right)\right\|_{\mathcal{S}(0, T)} \leq C\left\|u_{1}-u_{2}\right\|_{L^{2}(0, T)} .
$$

Thus, from now on, to emphasize the fact that the only varying variable of the weak solution of equation (37) is the bilinear multiplier $u$, we use the notation $y(u)$ where $y(u)$ satisfies

$$
\left\{\begin{array}{l}
y^{\prime \prime}(u)+A y(u)+\int_{0}^{t} k(t-s) A y(u) \mathrm{d} s+g(y(u))=u y(u)+f, \quad \text { in }(0, T), \\
y(u ; 0)=y_{0} \\
y^{\prime}(u ; 0)=y_{1} .
\end{array}\right.
$$

Furthermore, we present the following weakly continuous results to study the existence of the optimal pair in the subsequent section. For this, we need the following lemma.

Lemma 3 (see Simon [21]). Let $\mathscr{X}, \mathscr{Y}$, and $\mathscr{Z}$ be Banach spaces such that each embedding $\mathscr{X} \subset \mathscr{Y} \subset \mathscr{Z}$ is continuous and the embedding $\mathscr{X} \subset \mathcal{Y}$ is compact. Then, a bounded set of $W^{1, \infty}(0, T ; \mathscr{X}, \mathscr{Z})=\left\{g \mid g \in L^{\infty}(0, T ; \mathscr{X}), g^{\prime} \in L^{\infty}(0, T ; \mathscr{Z})\right\}$ is relatively compact in $C([0, T] ; \mathcal{Y})$.
Proposition 1. For fixed $\left(y_{0}, y_{1}, f\right) \in V \times H \times L^{2}(0, T ; H)$, the solution map from $u \in L^{2}(0, T)$ to $y(u) \in \mathscr{W}(0, T)$ of equation (37) is weakly continuous.

Proof. Let $u \in L^{2}(0, T)$, and let $\left\{u_{n}\right\} \subset L^{2}(0, T)$ be a bounded sequence such that

$$
u_{n} \longrightarrow u \text { weakly in } L^{2}(0, T), \quad \text { as } n \longrightarrow \infty \text {. }
$$

From now on, each state $y_{n}=y\left(u_{n}\right)$ is a solution of 


$$
\left\{\begin{array}{l}
y_{n}^{\prime \prime}+A y_{n}+\int_{0}^{t} k(t-s) A y_{n} \mathrm{~d} s+g\left(y_{n}\right)=u_{n} y_{n}+f, \quad \text { in }(0, T), \\
y_{n}(0)=y_{0} \\
y_{n}^{\prime}(0)=y_{1} .
\end{array}\right.
$$

From Theorem 1, we have

$$
\left\|y_{n}\right\|_{\mathcal{S}(0, T)} \leq C\left\|\left(y_{0}, y_{1}, f, u_{n}\right)\right\|_{\mathscr{P}}
$$

which implies that $y_{n}$ remains in a bounded set of $\mathscr{W}(0, T) \cap W^{1, \infty}(0, T ; V, H)$. Therefore, we can find a subsequence of $\left\{y_{n}\right\}$, say again $\left\{y_{n}\right\}$, and find $y \in \mathscr{W}(0, T)$ $\cap W^{1, \infty}(0, T ; V, H)$ such that

$$
y_{n} \longrightarrow y \text { weakly in } \mathscr{W}(0, T), \quad \text { as } n \longrightarrow \infty,
$$

$y_{n} \longrightarrow y$ weakly star in $L^{\infty}(0, T ; V), \quad$ as $n \longrightarrow \infty$,

$$
y_{n}^{\prime} \longrightarrow y^{\prime} \text { weakly star in } L^{\infty}(0, T ; H), \quad \text { as } n \longrightarrow \infty \text {. }
$$

Since the embedding $V \hookrightarrow H$ is compact, we can apply Lemma 3 to (42) and (43) with $\mathscr{X}=V$ and $\mathscr{Y}=\mathscr{Z}=H$ to verify that

$$
\left\{y_{n}\right\} \text { is a precompact set in } C([0, T] ; H) .
$$

Thus, we can find a subsequence of $\left\{y_{n}\right\}$, if necessary, still denoted by $\left\{y_{n}\right\}$, such that

$$
y_{n} \longrightarrow y \text { strongly in } C([0, T] ; H), \quad \text { as } n \longrightarrow \infty \text {. }
$$

For any given $\phi \in L^{2}(0, T ; V)$, using notation (27), we can deduce

$$
\begin{aligned}
\left|\left(g\left(y_{n}\right)-g(y), \phi\right)_{2}\right| & =(\gamma+1)\left|\left(B\left(y_{n}, y\right)\left(y_{n}-y\right), \phi\right)_{2}\right| \\
\leq & (\gamma+1)\left(\left(\left|y_{n}\right|+|y|\right)^{\gamma}\left|y_{n}-y\right|,|\phi|\right)_{2} \\
\leq & (\gamma+1)\left\|y_{n}-y\right\|_{2}\left\|\left(\left|y_{n}\right|+|y|\right)^{\gamma} \phi\right\|_{2} \\
\leq & C\left\|y_{n}-y\right\|_{2}\left\|\left|y_{n}\right|+|y|\right\|_{3 \gamma}^{\gamma}\|\phi\|_{6} \\
\leq & C\left\|y_{n}-y\right\|_{2}\left(\left\|y_{n}\right\|_{V}+\|y\|_{V}\right)^{\gamma}\|\phi\|_{V} \\
\leq & C\left(\left\|y_{n}\right\|_{C([0, T] ; V)}+\|y\|_{L^{\infty}(0, T ; V)}\right)^{\gamma} \\
& \left\|y_{n}-y\right\|_{2}\|\phi\|_{V} .
\end{aligned}
$$

Therefore, from (45) and (46), we can readily find a subsequence of $\left\{y_{n}\right\}$, if necessary, still denoted by itself, such that

$$
g\left(y_{n}\right) \longrightarrow g(y) \text { strongly in } L^{2}\left(0, T ; V^{\prime}\right), \quad \text { as } n \longrightarrow \infty .
$$

From (38) and (45), we may extract a subsequence of $\left\{u_{n}\right\}$, denoted again by $\left\{u_{n}\right\}$, such that

$$
u_{n} y_{n} \longrightarrow u y \text { weakly in } L^{2}(0, T ; H) \text {. }
$$

Replacing $y_{n}$ by $y_{n_{k}}$ in equation (39), if necessary, and letting $k \longrightarrow \infty$, we can conclude by the standard arguments as in Dautray and Lions ([20], pp.561-565) that the limit $y$ is a weak solution of

$$
\left\{\begin{array}{l}
y^{\prime \prime}+A y+\int_{0}^{t} k(t-s) A y \mathrm{~d} s+g(y)=u y+f, \quad \text { in }(0, T) \\
y(0)=y_{0} \\
y^{\prime}(0)=y_{1}
\end{array}\right.
$$

Moreover, by the uniqueness of the weak solutions, we can conclude that $y=y(u)$ in $\mathscr{W}(0, T)$, which implies that $y\left(u_{n}\right) \longrightarrow y(u)$ weakly in $\mathscr{W}(0, T)$.

This completes the proof.

\section{Quadratic Cost Minimax Control Problems}

In this section, we study the quadratic cost minimax optimal control problems for equation (52). Let the following be the set of the admissible controls:

$$
\mathcal{U}_{\mathrm{ad}}=\left\{c \in L^{2}(0, T) \mid c_{l} \leq c \leq c_{u} \text { a.e. in }[0, T]\right\},
$$

where $c_{l}$ and $c_{u}$ are lower and upper bounds of the control variables, respectively. Let the following be the set of the admissible disturbance or noises:

$$
\mathscr{V}_{\mathrm{ad}}=\left\{\eta \in L^{2}(0, T) \mid \eta_{l} \leq \eta \leq \eta_{u} \text { a.e. in }[0, T]\right\}
$$

where $\eta_{l}$ and $\eta_{u}$ are lower and upper bounds of the disturbance (noise) variables, respectively. For simplicity, let $\mathscr{F}_{\text {ad }}$ be a product space defined by $\mathscr{F}_{\text {ad }}=\mathscr{U}_{\text {ad }} \times \mathscr{V}_{\text {ad }}$.

Using Theorem 1, for fixed $\left(y_{0}, y_{1}, f\right) \in V \times$ $H \times L^{2}(0, T ; H)$, we can uniquely define the solution map $\mathscr{F}_{\text {ad }} \longrightarrow \mathcal{S}(0, T)$, which maps from $(c, \eta) \in \mathscr{F}_{\text {ad }}$ via $q=c+$ $\eta$ to the weak solution $y(q) \in \mathcal{S}(0, T)$, where $y(q)$ satisfies

$$
\left\{\begin{array}{l}
y^{\prime \prime}(q)+A y(q)+\int_{0}^{t} k(t-s) A y(q) \mathrm{d} s+g(y(q))=(c+\eta) y(q)+f, \quad \text { in }(0, T), \\
y(q ; 0)=y_{0}, \\
y^{\prime}(q ; 0)=y_{1} .
\end{array}\right.
$$


The weak solution $y(q)$ is called to be the state of the control system.

The quadratic cost function associated with control system (52) is given by

$$
\mathscr{J}(c, \eta)=\frac{1}{2}\left\|\mathscr{C} y(q)-Y_{d}\right\|_{\mathscr{M}}^{2}+\frac{\alpha}{2}\|c\|_{L^{2}(0, T)}^{2}-\frac{\beta}{2}\|\eta\|_{L^{2}(0, T)}^{2},
$$

where $\mathscr{M}$ is a Hilbert space of observation variables, $\mathscr{C} \in \mathscr{L}(\mathscr{W}(0, T), \mathscr{M})$ is a continuous linear operator, that is to say, observer, $Y_{d} \in \mathscr{M}$ is a desired value, and the positive constants $\alpha$ and $\beta$ are the relative weights of the second and third terms on the right-hand side of (53).

As indicated in the introduction, the minimax optimal control problem can be summarized as follows:

(i) Find an admissible control $c^{*} \in \mathcal{U}_{\text {ad }}$ and a disturbance (or noise) $\eta^{*} \in \mathscr{V}_{\text {ad }}$ such that $\left(c^{*}, \eta^{*}\right)$ is a saddle point of the functional $\mathscr{J}(c, \eta)$ of $(53)$, that is,

$$
\mathscr{J}\left(c^{*}, \eta\right) \leq \mathscr{J}\left(c^{*}, \eta^{*}\right) \leq \mathscr{J}\left(c, \eta^{*}\right), \quad \forall(c, \eta) \in \mathscr{F}_{\mathrm{ad}} .
$$

In this paper, we call such a pair $\left(c^{*}, \eta^{*}\right)$ in (54) as the optimal pair for the minimax optimal control problem with cost (53). To show the existence of the above saddle point $\left(c^{*}, \eta^{*}\right)$ (optimal pair), we need to show the solution map is differentiable in some sense. Then, with the assumptions on the weight constants $\alpha, \beta$ and the exponent $\gamma$, we utilize the arguments in Barbu and Precupanu [13] to show the existence of the optimal pairs. (ii) Characterize $\left(c^{*}, \eta^{*}\right)$ (optimality condition): in characterizing these optimal pairs, we introduce an appropriate adjoint system corresponding to the observed case and deduce the necessary optimal conditions through the variational inequality.

3.1. Differentiability of the Nonlinear Solution Map. In this section, we address the Fréchet differentiability of the nonlinear solution map, which is desirable for many applications.

For our study, we define the Fréchet differentiability of the nonlinear solution map as follows.

Definition 2. The solution map $u \longrightarrow y(u)$ of $L^{2}(0, T)$ into $\mathcal{S}(0, T)$ is said to be Fréchet differentiable on $L^{2}(0, T)$ if for any $u \in L^{2}(0, T)$, there exists $T(u) \in \mathscr{L}\left(L^{2}(0, T), \mathcal{S}(0, T)\right)$ such that, for any $w \in L^{2}(0, T)$,

$$
\frac{\|y(u+w)-y(u)-T(u) w\|_{\mathcal{S}(0, T)}}{\|w\|_{L^{2}(0, T)}} \longrightarrow 0, \quad \text { as }\|w\|_{L^{2}(0, T)} \longrightarrow 0 .
$$

The operator $T(u)$ is called the Fréchet derivative of $y$ at $u$, which we denote by $D y(u) . T(u) w=D y(u) w \in \mathcal{S}(0, T)$ is called the Fréchet derivative of $y$ at $u$ in the direction of $w \in L^{2}(0, T)$.

Theorem 2. The solution map $u \longrightarrow y(u)$ is Fréchet differentiable on $L^{2}(0, T)$, and the Fréchet derivative of $y(u)$ at $u$ in the direction $w \in L^{2}(0, T)$, that is to say $z=D y(u) w$, is given by the solution of

$$
\left\{\begin{array}{l}
z^{\prime \prime}+A z+\int_{0}^{t} k(t-s) A z \mathrm{~d} s+(\gamma+1)|y(u)|^{\gamma} z=u z+w y(u), \quad \text { in }(0, T) \\
z(0)=0 \\
z^{\prime}(0)=0
\end{array}\right.
$$

Proof. Let

$$
B(y(u)) z:=|y(u)|^{\gamma} z \text {. }
$$

Then, by (11) and Theorem 1, we can verify

$$
\begin{aligned}
\|B(y(u)) z\|_{2} & \leq\|y(u)\|_{3 \gamma}^{\gamma}\|z\|_{6} \\
& \leq C\|y(u)\|_{V}^{\gamma}\|z\|_{V} \\
& \leq C\|y(u)\|_{C([0, T] ; V)}^{\gamma}\|z\|_{V} \\
& \leq C\|y(u)\|_{\mathcal{S}(0, T)}^{\gamma}\|z\|_{V} \\
& \leq C\left\|\left(y_{0}, y_{1}, f, u\right)\right\|_{\mathscr{P}}^{\gamma}\|z\|_{V} .
\end{aligned}
$$

Hence, from (58), we see that

$$
B(y(u)) \in \mathscr{L}(V, H) \cap \mathscr{L}\left(H, V^{\prime}\right) .
$$

Since $w \in L^{2}(0, T)$ and $y(u) \in \mathcal{S}(0, T)$, we know that $w y(u) \in L^{2}(0, T ; H)$. By (59), we can use the results in [18] to verify that equation (56) admits a unique weak solution $z \in \mathcal{S}(0, T)$. And by Theorem 1, we can know that the weak solution $z(=z(w))$ of equation (56) satisfies

$$
\begin{aligned}
\|z(w)\|_{\mathcal{S}(0, T)} & \leq C\|w y(u)\|_{L^{2}(0, T ; H)} \\
& \leq C\|w\|_{L^{2}(0, T)}\|y(u)\|_{C(0, T ; H)} \\
& \leq C\|y(u)\|_{\mathcal{S}(0, T)}\|w\|_{L^{2}(0, T)} \\
& \leq C\left\|\left(y_{0}, y_{1}, f, u\right)\right\|_{\mathscr{P}}\|w\|_{L^{2}(0, T)} .
\end{aligned}
$$

Hence, from (60), the mapping $w \in L^{2} \quad(0, T) \mapsto z(w)$ $\in \mathcal{S}(0, T)$ is linear and bounded.

We set $\varphi=y(u+w)-y(u)-z$. Then, using notation (27) and noting 


$$
\begin{aligned}
& g(y(u+w))-g(y(u))-(\gamma+1)|y(u)|^{\gamma} z \\
= & (\gamma+1) B(y(u+w), y(u))(y(u+w) \\
& -y(u))-(\gamma+1)|y(u)|^{\gamma} z \\
= & (\gamma+1) B(y(u+w), y(u)) \varphi \\
& +(\gamma+1)\left(B(y(u+w), y(u))-|y(u)|^{\gamma}\right) z,
\end{aligned}
$$$$
\text { we know that } \varphi \text { satisfies }
$$

$$
\left\{\begin{array}{l}
\varphi^{\prime \prime}+A \varphi+\int_{0}^{t} k(t-s) A \varphi \mathrm{d} s+(\gamma+1) B(y(u+w), y(u)) \varphi=(u+w) \varphi+w z+I, \quad \text { in }(0, T) \\
\varphi(0)=0 \\
\varphi^{\prime}(0)=0
\end{array}\right.
$$

in the weak sense, where

$$
I=-(\gamma+1)\left(B(y(u+w), y(u))-|y(u)|^{\gamma}\right) z .
$$

Arguing as in the proof of Theorem 1, we can know that the weak solution $\varphi$ of equation (62) exists, and the following is fulfilled:

$$
\|\varphi\|_{\mathcal{S}(0, T)} \leq C\|w z+I\|_{L^{2}(0, T ; H)} .
$$

By (60), we can deduce

$$
\begin{aligned}
\|w z\|_{L^{2}(0, T ; H)} & \leq\|w\|_{L^{2}(0, T)}\|z\|_{C([0, T] ; H)} \\
& \leq C\|w\|_{L^{2}(0, T)}\|z\|_{\mathcal{S}(0, T)} \\
& \leq C\|w\|_{L^{2}(0, T)}^{2}, \\
& \left\|\left(B(y(u+w), y(u))-|y(u)|^{\gamma}\right) z\right\|_{2} \\
& \leq\left\|B(y(u+w), y(u))-|y(u)|^{\gamma}\right\|_{3}\|z\|_{6} \\
& \leq C\left\|B(y(u+w), y(u))-|y(u)|^{\gamma}\right\|_{3}\|z\|_{V} \\
& \leq C\left\|B(y(u+w), y(u))-|y(u)|^{\gamma}\right\|_{3}\|z\|_{C([0, T] ; V)} \\
& \leq C\|z\|_{\mathcal{S}(0, T)}\left\|B(y(u+w), y(u))-|y(u)|^{\gamma}\right\|_{3} \\
& \leq C\|w\|_{L^{2}(0, T)}\left\|B(y(u+w), y(u))-|y(u)|^{\gamma}\right\|_{3} .
\end{aligned}
$$

By Theorem 1, we can see that

$$
\|y(u+w)-y(u)\|_{\mathcal{S}(0, T)} \leq C\|w\|_{L^{2}(0, T)} .
$$

Thus, if $\|w\|_{L^{2}(0, T)} \longrightarrow 0$, then by (66), we can extract a subsequence, still denoted by $\{y(u+w)\}$, such that

$$
B(y(u+w), y(u))-|y(u)|^{\gamma} \longrightarrow 0, \quad \text { a.e. in } Q \text {. }
$$

And from (11), we know that

$$
\begin{aligned}
\left.\left.|B(y(u+w), y(u))-| y(u)\right|^{\gamma}\right|^{3} & \leq 8(|y(u+w)|+|y(u)|)^{3 \gamma} \\
& \in L^{1}(Q) .
\end{aligned}
$$

Thus, by the Lebesgue dominated convergence theorem through (67) and (68), we can obtain

$$
\left\|B(y(u+w), y(u))-|y(u)|^{\gamma}\right\|_{L^{3}(Q)} \longrightarrow 0 \text { as }\|w\|_{L^{2}(0, T)} \longrightarrow 0 .
$$

Hence, from (64) to (69), we can obtain

$$
\begin{aligned}
\|\varphi\|_{\mathcal{S}(0, T)} & \leq C\|w z+I\|_{L^{2}(0, T ; H)} \\
& \leq C\left(\|w z\|_{L^{2}(0, T ; H)}+\|I\|_{L^{2}(0, T ; H)}\right) \\
& \leq C\|w\|_{L^{2}(0, T)}\left(\|w\|_{L^{2}(0, T)}+\left\|B(y(u+w), y(u))-|y(u)|^{\gamma}\right\|_{L^{3}(Q)}\right),
\end{aligned}
$$

which immediately implies that $\|\varphi\|_{\mathcal{S}(0, T)}=o\left(\|w\|_{L^{2}(0, T)}\right)$ as $\|w\|_{L^{2}(0, T)} \longrightarrow 0$.

This completes the proof.

As before, for simplicity, we denote $c+\eta$ and $c^{*}+\eta^{*}$ by $q$ and $q^{*}$, respectively. From Theorem 2, we know that the map $(c, \eta) \longrightarrow y(q)$ of $\mathscr{F}_{\text {ad }}$ to $\mathcal{S}(0, T)$ is Fréchet differentiable at $\left(c^{*}, \eta^{*}\right)$, and the Fréchet derivative of $y(q)$ at $\left(c^{*}, \eta^{*}\right)$ in the direction $w=(h, l) \in\left[L^{2}(0, T)\right]^{2}$, say $z=D y\left(q^{*}\right) w$, is a unique weak solution of the following problem: 


$$
\left\{\begin{array}{l}
z^{\prime \prime}+A z+\int_{0}^{t} k(t-s) A z \mathrm{~d} s+(\gamma+1)\left|y\left(q^{*}\right)\right|^{\gamma} z=q^{*} z+(h+l) y\left(q^{*}\right), \quad \text { in }(0, T) \\
z(0)=0 \\
z^{\prime}(0)=0 .
\end{array}\right.
$$

The following results will be used in showing the existence of optimal pairs.

Proposition 2. Assume that the exponent $\gamma \geq 1$. For given $w \in L^{2}(0, T)$, the Fréchet derivative $D y(u) w$ is locally Lipschitz continuous on $L^{2}(0, T)$. Indeed, it is satisfied that

$$
\left\|D y\left(u_{1}\right) w-D y\left(u_{2}\right) w\right\|_{\mathcal{S}(0, T)} \leq C\left\|u_{1}-u_{2}\right\|_{L^{2}(0, T)}\|w\|_{L^{2}(0, T)} \text {, }
$$

where $C>0$ is a constant depending on the data.

Proof. Let $z_{i}=D y\left(u_{i}\right) w(i=1,2)$ be the weak solutions of equation (56) corresponding to $u_{i}(i=1,2)$. If we set $\phi=z_{1}-z_{2}$, then we can know that $\phi$ satisfies

$$
\left\{\begin{array}{l}
\phi^{\prime \prime}+A \phi+\int_{0}^{t} k(t-s) A \phi \mathrm{d} s+(\gamma+1)\left|y\left(u_{1}\right)\right|^{\gamma} \phi=u_{1} \phi+I_{1}+I_{2}, \quad \text { in }(0, T) \\
\phi(0)=0 \\
\phi^{\prime}(0)=0
\end{array}\right.
$$

in the weak sense, where

$$
\begin{aligned}
& I_{1}=-\gamma(\gamma+1) \int_{0}^{1}\left(\theta\left|y\left(u_{1}\right)\right|+(1-\theta)\left|y\left(u_{2}\right)\right|\right)^{(\gamma-1)} \mathrm{d} \theta\left(\left|y\left(u_{1}\right)\right|-\left|y\left(u_{2}\right)\right|\right) z_{2}, \\
& I_{2}=\left(u_{1}-u_{2}\right) z_{2}+w\left(y\left(u_{1}\right)-y\left(u_{2}\right)\right) .
\end{aligned}
$$

As before, arguing as in the proof of Theorem 1, we can deduce that

$$
\|\phi\|_{\mathcal{S}(0, T)} \leq C\left\|I_{1}+I_{2}\right\|_{L^{2}(0, T ; H)}
$$

In estimating $I_{1}$, the case of $\gamma=1$ will be clarified as we will see later. Thus, we restrict to the case of $\gamma>1$ to estimate $I_{1}$. By (11), Theorem 1, and (60), we can obtain

$$
\begin{aligned}
\left\|I_{1}\right\|_{2} \leq & \gamma(\gamma+1)\left\|\left(\left|y\left(u_{1}\right)\right|+\left|y\left(u_{2}\right)\right|\right)^{\gamma-1}\left|y\left(u_{1}\right)-y\left(u_{2}\right)\right|_{2}\right\|_{2} \\
& \leq C\left\|\left(\left|y\left(u_{1}\right)\right|+\left|y\left(u_{2}\right)\right|\right)^{\gamma-1}\right\|_{6}\left\|y\left(u_{1}\right)-y\left(u_{2}\right)\right\|_{6}\left\|_{2}\right\|_{6} \\
& \leq C\left(\left\|y\left(u_{1}\right)\right\|_{V}+\left\|y\left(u_{2}\right)\right\|_{V}\right)^{\gamma-1}\left\|y\left(u_{1}\right)-y\left(u_{2}\right)\right\|_{V}\left\|_{2}\right\|_{V} \\
& \leq C\left(\left\|y\left(u_{1}\right)\right\|_{C([0, T] ; V)}+\left\|y\left(u_{2}\right)\right\|_{C([0, T] ; V)}\right)^{\gamma-1}\left\|y\left(u_{1}\right)-y\left(u_{2}\right)\right\|_{C([0, T] ; V)}\left\|z_{2}\right\|_{C([0, T] ; V)} \\
& \leq C\left\|u_{1}-u_{2}\right\|_{L^{2}(0, T)}\|w\|_{L^{2}(0, T)}, \\
\left\|I_{2}\right\|_{L^{2}(0, T ; H)} \leq & \left\|\left(u_{1}-u_{2}\right) z_{2}\right\|_{L^{2}(0, T ; H)}+\left\|w\left(y\left(u_{1}\right)-y\left(u_{2}\right)\right)\right\|_{L^{2}(0, T ; H)} \\
\leq & \left\|u_{1}-u_{2}\right\|_{L^{2}(0, T)}\left\|z_{2}\right\|_{C([0, T] ; H)}+\|w\|_{L^{2}(0, T)}\left\|y\left(u_{1}\right)-y\left(u_{2}\right)\right\|_{C([0, T] ; H)} \\
\leq & C\left(\left\|u_{1}-u_{2}\right\|_{L^{2}(0, T)}\left\|z_{2}\right\|_{\mathcal{\delta}(0, T)}\right. \\
& \left.+\|w\|_{L^{2}(0, T)}\left\|y\left(u_{1}\right)-y\left(u_{2}\right)\right\|_{\mathcal{S}(0, T)}\right) \\
\leq & C\left\|u_{1}-u_{2}\right\|_{L^{2}(0, T)}\|w\|_{L^{2}(0, T)} .
\end{aligned}
$$


From (75) to (77), we can get the following:

$$
\|\phi\|_{\mathcal{S}(0, T)} \leq C\left\|u_{1}-u_{2}\right\|_{L^{2}(0, T)}\|w\|_{L^{2}(0, T)} .
$$

This completes the proof.

3.2. Existence of the Optimal Pairs. By the minimax theorem in infinite dimensions in Barbu and Precupanu [13], we study the existence of the optimal pairs in the underlying control system with quadratic cost function (53).

Theorem 3. Assume that the exponent $\gamma \geq 1$ in control system equation (52). Then, for sufficiently large $\alpha$ and $\beta$ in (53), there exists $\left(c^{*}, \eta^{*}\right) \in \mathscr{F}_{\text {ad }}$ satisfying (54).

In order to prove this theorem, we mainly illustrate the following as in [8] (cf. [9]):

(i) For sufficiently large $\alpha$ and $\beta$ in (53), the maps $c \longrightarrow \mathscr{J}(c, \eta)$ and $\eta \longrightarrow \mathscr{J}(c, \eta)$ are convex and concave for all $\eta \in \mathscr{V}_{\text {ad }}$ and for all $c \in \mathcal{U}_{a d}$, respectively (ii) The maps $c \longrightarrow \mathscr{J}(c, \eta)$ and $\eta \longrightarrow \mathscr{J}(c, \eta)$ are lower and upper semicontinuous for all $\eta \in \mathscr{V}_{\text {ad }}$ and for all $c \in \mathcal{U}_{a d}$, respectively

Proof

(i) Since the map $c \longrightarrow y(c+\eta)$ where $\eta$ is fixed is Fréchet differentiable, the map $c \longrightarrow \mathscr{J}(c, \eta)$ is also Fréchet differentiable. Let $D_{c} \mathscr{J}\left(c_{1}, \eta\right) \quad\left(c_{1}-c_{2}\right)$ and $D_{c} \mathscr{J}\left(c_{2}, \eta\right)\left(c_{1}-c_{2}\right)$ be the Fréchet derivatives of the map $c \longrightarrow \mathscr{J}(c, \eta)$ at $c_{1}$ and $c_{2}$ in the direction $c_{1}-c_{2}$, respectively, where $\eta$ holds fixed. To verify the convexity of the map $c \longrightarrow \mathscr{J}(c, \eta)$, it is sufficient to show that

$\left(D_{c} \mathscr{J}\left(c_{1}, \eta\right)-D_{c} \mathscr{J}\left(c_{2}, \eta\right)\right)\left(c_{1}-c_{2}\right) \geq 0, \quad \forall c_{1}, c_{2} \in \mathcal{U}_{\mathrm{ad}}$,

for any fixed $\eta \in \mathscr{V}_{\text {ad }}$. (79) means

$$
\begin{aligned}
\left(\mathscr{C} y\left(c_{1}+\eta\right)-Y_{d},\right. & \left.\mathscr{C D}_{c} y\left(c_{1}+\eta\right)\left(c_{1}-c_{2}\right)\right)_{\mathscr{M}}+\alpha\left(c_{1}, c_{1}-c_{2}\right)_{L^{2}(0, T)} \\
& -\left(\mathscr{C} y\left(c_{2}+\eta\right)-Y_{d}, \mathscr{C D}_{c} y\left(c_{2}+\eta\right)\left(c_{1}-c_{2}\right)\right)_{\mathscr{M}} \\
& -\alpha\left(c_{2}, c_{1}-c_{2}\right)_{L^{2}(0, T)} \geq 0, \quad \forall c_{1}, c_{2} \in \mathscr{U}_{\mathrm{ad}},
\end{aligned}
$$

where $D_{c} y\left(c_{i}+\eta\right)\left(c_{1}-c_{2}\right)(i=1,2)$ are weak solutions of equation (71), in which $q^{*} z+(h+l) y(p)$ is replaced by $\left(c_{i}+\eta\right) z+\left(c_{1}-c_{2}\right) y\left(c_{i}+\eta\right)(i=1,2)$, respectively. We can deduce that $(80)$ is equivalent to

$$
\begin{aligned}
& \left(\mathscr{C}\left(y\left(c_{1}+\eta\right)-y\left(c_{2}+\eta\right)\right), \mathscr{C} D_{c} y\left(c_{1}+\eta\right)\left(c_{1}-c_{2}\right)\right)_{\mathscr{M}} \\
& +\left(\mathscr{C} y\left(c_{2}+\eta\right)-Y_{d}, \mathscr{C}\left(D_{c} y\left(c_{1}+\eta\right)\left(c_{1}-c_{2}\right)-D_{c} y\left(c_{2}+\eta\right)\left(c_{1}-c_{2}\right)\right)\right)_{\mathscr{M}} \\
& +\alpha\left\|c_{1}-c_{2}\right\|_{L^{2}(0, T)}^{2} \geq 0, \quad \forall c_{1}, c_{2} \in \mathscr{U}_{\mathrm{ad}} .
\end{aligned}
$$

By Theorem 1, (60), and Proposition 2, we can have the following estimations:

$$
\begin{aligned}
&\left|\left(\mathscr{C}\left(y\left(c_{1}+\eta\right)-y\left(c_{2}+\eta\right)\right), \mathscr{C} D_{c} y\left(c_{1}, \eta\right)\left(c_{1}-c_{2}\right)\right)_{\mathscr{M}}\right| \\
& \leq\left\|\mathscr{C}\left(y\left(c_{1}+\eta\right)-y\left(c_{2}+\eta\right)\right)\right\|_{\mathscr{M}}\left\|\mathscr{C} D_{c} y\left(c_{1}+\eta\right)\left(c_{1}-c_{2}\right)\right\|_{\mathscr{M}} \\
& \leq C\left\|y\left(c_{1}+\eta\right)-y\left(c_{2}+\eta\right)\right\|_{\mathcal{S}(0, T)}\left\|D_{c} y\left(c_{1}+\eta\right)\left(c_{1}-c_{2}\right)\right\|_{\mathcal{S}(0, T)} \\
& \leq C\left\|c_{1}-c_{2}\right\|_{L^{2}(0, T)}^{2}, \\
&\left.\leq \| \mathscr{C} y\left(c_{2}+\eta\right)-Y_{d}, \mathscr{C}\left(D_{c} y\left(c_{1}+\eta\right)\left(c_{1}-c_{2}\right)-D_{c} y\left(c_{2}+\eta\right)\left(c_{1}-c_{2}\right)\right)\right)_{\mathscr{M}}\left\|_{d}\right\|_{\mathscr{M}}\left\|\mathscr{C}\left(D_{c} y\left(c_{1}+\eta\right)\left(c_{1}-c_{2}\right)-D_{c} y\left(c_{2}+\eta\right)\left(c_{1}-c_{2}\right)\right)\right\|_{\mathscr{M}} \\
& \leq C\left(\left\|\mathscr{C} y\left(c_{2}+\eta\right)\right\|_{\mathscr{M}}+\left\|Y_{d}\right\|_{\mathscr{M}}\right) \times\left\|D_{c} y\left(c_{1}+\eta\right)\left(c_{1}-c_{2}\right)-D_{c} y\left(c_{2}+\eta\right)\left(c_{1}-c_{2}\right)\right\|_{\mathcal{S}(0, T)} \\
& \leq C\left(\left\|\mathscr{C} y\left(c_{2}+\eta\right)\right\|_{\mathscr{M}}+\left\|Y_{d}\right\|_{\mathscr{M}}\right)\left\|c_{1}-c_{2}\right\|_{L^{2}(0, T)}^{2} \\
& \leq C\left\|c_{1}-c_{2}\right\|_{L^{2}(0, T)}^{2} .
\end{aligned}
$$


From (81) to (83), we can verify that there exists sufficiently large $\alpha_{l}\left(\mathscr{P}, \mathscr{F}_{\text {ad }}, Y_{d}, \mathscr{C}, \mathscr{M}\right)$ such that, for any $\alpha>\alpha_{l}\left(\mathscr{P}, \mathscr{F}_{\text {ad }}, Y_{d}, \mathscr{C}, \mathscr{M}\right)$, inequality (79) holds. Thus, the map $c \longrightarrow \mathscr{J}(c, \eta)$ is convex.

Similarly, we can show that there exists sufficiently large $\beta_{l}\left(\mathscr{P}, \mathscr{F}_{\text {ad }}, Y_{d}, \mathscr{C}, \mathscr{M}\right)$ such that the following inequality

$$
\left(D_{\eta} \mathscr{F}\left(c, \eta_{1}\right)-D_{\eta} \mathscr{F}\left(c, \eta_{2}\right)\right)\left(\eta_{1}-\eta_{2}\right) \leq 0, \quad \forall \eta_{1}, \eta_{2} \in \mathscr{V}_{\text {ad }} \text {, }
$$

is satisfied for any fixed $c \in \mathscr{U}_{\text {ad }}$ and $\beta>\beta_{l}\left(\mathscr{P}, \mathscr{F}_{\text {ad }}, Y_{d}, \mathscr{C}, \mathscr{M}\right)$. This also indicates the concavity of the map $\eta \longrightarrow \mathscr{J}(c, \eta)$.

(ii) Since $\left\{c_{n}\right\}$ is bounded in $L^{2}(0, T)$, we can extract a subsequence $\left\{c_{n_{k}}\right\} \subset\left\{c_{n}\right\}$ such that

$$
c_{n_{k}} \longrightarrow c^{*} \text { weakly in } L^{2}(0, T), \quad \text { as } k \longrightarrow \infty \text {. }
$$

Then, since $\mathscr{C}$ is a continuous linear operator on $\mathscr{W}(0, T)$, by Proposition 1 , we obtain

$$
\mathscr{C} y\left(c_{n_{k}}+\eta\right) \longrightarrow \mathscr{C} y\left(c^{*}+\eta\right) \text { weakly in } \mathscr{M}, \quad \text { as } k \longrightarrow \infty \text {, }
$$

$\forall \eta \in \mathscr{V}_{\text {ad }}$. Since the norm is weakly lower semicontinuous, we can see from (85) and (86) that the map $c \longrightarrow \mathscr{J}(c, \eta)$ is lower semicontinuous for all $\eta \in \mathscr{V}_{\text {ad }}$. By similar arguments, we can prove that the map $\eta \longrightarrow \mathscr{J}(c, \eta)$ is upper semicontinuous for all $c \in \mathscr{U}_{\mathrm{ad}}$.

Next, we prove the existence of an optimal pair $\left(c^{*}, \eta^{*}\right) \in \mathscr{F}_{\text {ad }}$ : let $\left\{c_{n}\right\} \subset \mathcal{U}_{\text {ad }}$ be a minimizing sequence of $\mathscr{J}(c, \eta)$ where $\eta \in \mathscr{V}_{\text {ad }}$ is fixed. Thus,

$$
\liminf _{n \longrightarrow \infty} \mathscr{J}\left(c_{n}, \eta\right)=\inf _{c \in \mathscr{U}_{a d}} \mathscr{J}(c, \eta) .
$$

Then, by (i) and (ii), we know that

$$
\mathscr{J}_{0}(\eta)=\liminf _{n \longrightarrow \infty} \mathscr{J}\left(c_{n}, \eta\right) \geq \mathscr{J}\left(c^{*}, \eta\right), \quad \forall \eta \in \mathscr{V}_{\text {ad }},
$$

but since $\mathscr{f}_{0}(\eta) \leq \mathscr{J}\left(c^{*}, \eta\right)$, we have

$$
\mathscr{F}_{0}(\eta)=\mathscr{F}\left(c^{*}, \eta\right)=\inf _{c \in \mathscr{U}_{\mathrm{ad}}} \mathscr{f}(c, \eta), \quad \forall \eta \in \mathscr{V}_{\mathrm{ad}} .
$$
that

Similarly, we also know that there exists $\eta^{*} \in \mathscr{V}_{\text {ad }}$ such

$$
\mathscr{J}_{0}\left(\eta^{*}\right)=\sup _{\eta \in \mathscr{V}_{\text {ad }}} \mathscr{F}_{0}(\eta) .
$$

From (89) and (90), we can conclude that $\left(c^{*}, \eta^{*}\right) \in \mathscr{F}_{\text {ad }}$ is an optimal pair for cost (53).

This completes the proof.

3.3. Necessary Conditions of Optimal Pairs. In this section, we study the necessary optimality conditions that have to be satisfied by each optimal pair of the minimax optimal control problem with cost (53) in which the following two types of observations are considered:

(1) We take $\mathscr{M}_{1}=L^{2}(0, T ; H) \times H \quad$ and $\mathscr{C}_{1} \in \mathscr{L}\left(\mathscr{W}(0, T), \mathscr{M}_{1}\right)$ and observe $\mathscr{C}_{1} y(q)=(y(q), y(q ; T)) \in L^{2}(0, T ; H) \times H$

(2) We take $\mathscr{M}_{2}=L^{2}(0, T ; H)$ and $\mathscr{C}_{2} \in \mathscr{L}(\mathscr{W}(0, T)$, $\left.\mathscr{M}_{2}\right)$ and observe $\mathscr{C}_{2} y(q)=y^{\prime}(q) \in L^{2}(0, T ; H)$

Since $y(q) \in \mathscr{W}(0, T) \subset C([0, T] ; H)$, the above observations are meaningful.

3.3.1. Case of Distributive and Terminal Values' Observations $\mathscr{C}_{1} \in \mathscr{L}\left(\mathscr{W}(0, T), \mathscr{M}_{1}\right)$. We consider the cost functional expressed by

$$
\begin{aligned}
\mathscr{J}(c, \eta)= & \frac{1}{2}\left\|y(q)-y_{d}\right\|_{L^{2}(0, T ; H)}^{2}+\frac{1}{2}\left\|y(q ; T)-y_{d}^{T}\right\|_{2}^{2} \\
& +\frac{\alpha}{2}\|c\|_{L^{2}(0, T)}^{2}-\frac{\beta}{2}\|\eta\|_{L^{2}(0, T)}^{2},
\end{aligned}
$$

where $y_{d} \in L^{2}(0, T ; H)$ and $y_{d}^{T} \in H$ are desired values. Let $\left(c^{*}, \eta^{*}\right) \in\left[L^{2}(0, T)\right]^{2}\left(q^{*}=c^{*}+\eta^{*}\right)$ be an optimal pair subject to equation (52) and quadratic cost (91). Now, we are about to formulate the following adjoint equation corresponding to cost (91) and equation (52) in which $(c, \eta)$ with $q=c+\eta$ is replaced by $\left(c^{*}, \eta^{*}\right)$ with $q^{*}=c^{*}+\eta^{*}$ :

$$
\left\{\begin{array}{l}
p^{\prime \prime}+A p+\int_{t}^{T} k(\sigma-t) A p \mathrm{~d} \sigma+(\gamma+1)\left|y\left(q^{*}\right)\right|^{\gamma} p=\left(c^{*}+\eta^{*}\right) p+y\left(q^{*}\right)-y_{d}, \quad \text { in }(0, T), \\
p(T)=0 \\
p^{\prime}(T)=-y\left(q^{*} ; T\right)+y_{d}^{T}
\end{array}\right.
$$

Proposition 3. Equation (92) admits a unique weak solution $p \in \mathcal{S}(0, T)$.

Proof. From the observation conditions $y\left(q^{*}\right)-y_{d}$ $\in L^{2}(0, T ; H)$ and $y\left(q^{*} ; T\right)-y_{d}^{T} \in H$ and (59), we can refer to the results in [18] (cf. Dautray and Lions [20], pp.655-659) to ensure that equation (92), after reversing the direction of time $t \longrightarrow T-t$, admits a unique weak solution $p \in \mathcal{S}(0, T)$. This completes the proof.

We now discuss the first-order optimality conditions for minimax optimal control problem (54) for quadratic cost function (91). 
Theorem 4. If $\alpha$ and $\beta$ in cost (91) are large enough and the exponent $\gamma \geq 1$, then an optimal control $c^{*} \in \mathscr{U}_{\text {ad }}$ and $a$ disturbance $\eta^{*} \in \mathscr{V}_{a d}$, namely, an optimal pair $\left(c^{*}, \eta^{*}\right) \in$ $\mathscr{F}_{a d}$ satisfying (54), can be given by

$$
\begin{aligned}
& c^{*}=\max \left\{c_{l}, \min \left\{-\frac{\left(y\left(q^{*}\right), p\right)_{2}}{\alpha}, c_{u}\right\}\right\}, \\
& \eta^{*}=\max \left\{\eta_{l}, \min \left\{\frac{\left(y\left(q^{*}\right), p\right)_{2}}{\beta}, \eta_{u}\right\}\right\},
\end{aligned}
$$

where $p$ is the weak solution of equation (92).

Proof. By the assumptions of Theorem 4, we can verify through Theorem 3 that there exists an optimal pair in (54) with cost (91). Let $\left(c^{*}, \eta^{*}\right) \in \mathscr{F}_{\text {ad }}$ be an optimal pair in (54) with cost (91) and $y\left(q^{*}\right)$ be the corresponding weak solution of equation (52).
Due to Theorem 2, we know that the map $(c, \eta) \longrightarrow y(q)$ is Fréchet differentiable at $\left(c^{*}, \eta^{*}\right)$ in the direction $w=(h, l) \in\left[L^{2}(0, T)\right]^{2}$ which satisfies for sufficiently small $\epsilon>0$ that $\left(c^{*}+\varepsilon h, \eta^{*}+\varepsilon l\right) \in \mathscr{F}_{\text {ad }}$. Thus, the map $(c, \eta) \longrightarrow y(q)$ is also (strongly) Gâteaux differentiable at $\left(c^{*}, \eta^{*}\right)$ in the direction $(h, l) \in\left[L^{2}(0, T)\right]^{2}$. Indeed, we have

$$
\frac{y\left(q^{*}+\varepsilon(h+l)\right)-y\left(q^{*}\right)}{\varepsilon} \longrightarrow z(=z(w)) \text { strongly in } \mathcal{S}(0, T),
$$

as $\varepsilon \longrightarrow 0^{+}$, where $z=D y\left(q^{*}\right) w$ is a unique solution of equation (71). Therefore, we can obtain from (94) the Gâteaux derivative of cost $(91)$ at $\left(c^{*}, \eta^{*}\right)$ in the direction $w=(h, l)$ as follows:

$$
\begin{aligned}
D \mathscr{J}\left(c^{*}, \eta^{*}\right)(h, l)= & \lim _{\varepsilon \longrightarrow 0^{+}} \frac{\mathscr{J}\left(c^{*}+\varepsilon h, \eta^{*}+\varepsilon l\right)-\mathscr{J}\left(c^{*}, \eta^{*}\right)}{\varepsilon} \\
= & \lim _{\varepsilon \longrightarrow 0^{+}} \frac{1}{2} \int_{0}^{T}\left(y\left(q^{*}+\varepsilon(h+l)\right)+y\left(q^{*}\right)-2 y_{d}, \frac{y\left(q^{*}+\varepsilon(h+l)\right)-y\left(q^{*}\right)}{\varepsilon}\right)_{2} \mathrm{~d} t \\
& +\lim _{\varepsilon \longrightarrow 0^{+}} \frac{1}{2}\left(y\left(q^{*}+\varepsilon(h+l) ; T\right)+y\left(q^{*} ; T\right)-2 y_{d}^{T}, \frac{y\left(q^{*}+\varepsilon(h+l) ; T\right)-y\left(q^{*} ; T\right)}{\varepsilon}\right)_{2} \\
& +\lim _{\varepsilon \longrightarrow 0^{+}}\left[\frac{\alpha}{2}\left(2\left(c^{*}, h\right)_{L^{2}(0, T)}+\varepsilon\|h\|_{L^{2}(0, T)}^{2}\right)-\frac{\beta}{2}\left(2\left(\eta^{*}, l\right)_{L^{2}(0, T)}+\varepsilon\|l\|_{L^{2}(0, T)}^{2}\right)\right] \\
= & \int_{0}^{T}\left(y\left(q^{*}\right)-y_{d}, z\right)_{2} \mathrm{~d} t+\left(y\left(q^{*} ; T\right)-y_{d}^{T}, z(T)\right)_{2} \\
& +\alpha\left(c^{*}, h\right)_{L^{2}(0, T)}-\beta\left(\eta^{*}, l\right)_{L^{2}(0, T)},
\end{aligned}
$$

where $z=D y\left(q^{*}\right) w$ is a solution of equation (71).

We multiply both sides of the weak form of equation (92) by $z$ which is a solution of equation (71) and integrate it over $[0, T]$. Then, we obtain

$$
\begin{aligned}
& \int_{0}^{T}\left\langle p^{\prime \prime}+A p, z\right\rangle_{V^{\prime}, V} \mathrm{~d} t+\int_{0}^{T}\left\langle\int_{t}^{T} k(\sigma-t) A p \mathrm{~d} \sigma, z\right\rangle_{V^{\prime}, V} \mathrm{~d} t \\
& +\int_{0}^{T}\left((\gamma+1)\left|y\left(q^{*}\right)\right|^{\gamma} p-q^{*} p, z\right)_{2} \mathrm{~d} t \\
= & \int_{0}^{T}\left(y\left(q^{*}\right)-y_{d}, z\right)_{2} \mathrm{~d} t .
\end{aligned}
$$

By integration by parts, the terminal value of the weak solution $p$ of equation (92), and Fubini's theorem, (96) can be written again as

$$
\begin{aligned}
& \int_{0}^{T}\left\langle p, z^{\prime \prime}+A z\right\rangle_{V, V^{\prime}} \mathrm{d} t-\left(y\left(q^{*} ; T\right)-y_{d}^{T}, z(T)\right)_{2} \\
& +\int_{0}^{T}\left\langle p, \int_{0}^{t} k(t-s) A z \mathrm{~d} s\right\rangle_{V, V^{\prime}} \mathrm{d} t \\
& +\int_{0}^{T}\left(p,(\gamma+1)\left|y\left(q^{*}\right)\right|^{\gamma} z\right)_{2} \mathrm{~d} t-\int_{0}^{T}\left(p, q^{*} z\right)_{2} \mathrm{~d} t \\
= & \int_{0}^{T}\left(y\left(q^{*}\right)-y_{d}, z\right)_{2} \mathrm{~d} t .
\end{aligned}
$$

Since $z$ is the solution of equation (71), by (97), we can deduce the following:

$$
\begin{aligned}
& \int_{0}^{T}\left(y\left(q^{*}\right)-y_{d}, z\right)_{2} \mathrm{~d} t+\left(y\left(q^{*} ; T\right)-y_{d}^{T}, z(T)\right)_{2} \\
= & \left(h+l,\left(y\left(q^{*}\right), p\right)_{2}\right)_{L^{2}(0, T)} .
\end{aligned}
$$

Therefore, from (95) and (98), we can obtain that 


$$
\begin{aligned}
\operatorname{DJ}\left(c^{*}, \eta^{*}\right)(h, l)= & \left(\alpha c^{*}+\left(y\left(q^{*}\right), p\right)_{2}, h\right)_{L^{2}(0, T)} \\
& +\left(-\beta \eta^{*}+\left(y\left(q^{*}\right), p\right)_{2}, l\right)_{L^{2}(0, T)} .
\end{aligned}
$$

Since $\left(c^{*}, \eta^{*}\right) \in \mathscr{F}_{\text {ad }}$ is an optimal pair in (54), we know

$$
\begin{aligned}
& D_{c} J\left(c^{*}, \eta^{*}\right)(h) \geq 0, \\
& D_{\eta} J\left(c^{*}, \eta^{*}\right)(l) \leq 0, \quad(h, l) \in\left[L^{2}(0, T)\right]^{2} .
\end{aligned}
$$

Therefore, we can obtain the following from (99) and (100):

$$
\begin{array}{r}
\left(\alpha c^{*}+\left(y\left(q^{*}\right), p\right)_{2}, h\right)_{L^{2}(0, T)} \geq 0, \\
\left(-\beta \eta^{*}+\left(y\left(q^{*}\right), p\right)_{2}, l\right)_{L^{2}(0, T)} \leq 0,
\end{array}
$$

where $(h, l) \in\left[L^{2}(0, T)\right]^{2}$. By considering the signs of the variations $h$ and $l$ in (101), which depend on $c^{*}$ and $\eta^{*}$, respectively, we can deduce from (101) that

$$
\begin{aligned}
& c^{*}=\max \left\{c_{l}, \min \left\{-\frac{\left(y\left(q^{*}\right), p\right)_{2}}{\alpha}, c_{u}\right\}\right\}, \\
& \eta^{*}=\max \left\{\eta_{l}, \min \left\{\frac{\left(y\left(q^{*}\right), p\right)_{2}}{\beta}, \eta_{u}\right\}\right\} .
\end{aligned}
$$

This completes the proof.

3.3.2. Case of Velocity's Distributive Value Observation $\mathscr{C}_{2} \in \mathscr{L}\left(\mathscr{W}(0, T), \mathscr{M}_{2}\right)$. In this case, we consider the following cost functional:

$$
\mathscr{f}(c, \eta)=\frac{1}{2}\left\|y^{\prime}(q)-y_{d}\right\|_{L^{2}(0, T ; H)}^{2}+\frac{\alpha}{2}\|c\|_{L^{2}(0, T)}^{2}-\frac{\beta}{2}\|\eta\|_{L^{2}(0, T)}^{2},
$$

where $y_{d} \in L^{2}(0, T ; H)$ is the desired value. Let $\left(c^{*}, \eta^{*}\right) \in \mathscr{F}_{\text {ad }}$ be an optimal pair subject to equation (52) and quadratic cost (103). In this case, we introduce the following adjoint equation corresponding to cost (103) and equation (52) in which $q=c+\eta$ is replaced by $q^{*}=c^{*}+\eta^{*}$ :

$$
\left\{\begin{array}{l}
p^{\prime \prime}+A p+\int_{t}^{T} k(0) A p \mathrm{~d} s+\int_{t}^{T} \int_{s}^{T} k^{\prime}(\sigma-s) A p \mathrm{~d} \sigma \mathrm{d} s-(\gamma+1) \int_{t}^{T}\left|y\left(q^{*}\right)\right|^{\gamma} p^{\prime} d+\int_{t}^{T} q^{*} p^{\prime} \mathrm{d} s=y^{\prime}\left(q^{*}\right)-y_{d}, \quad \text { in }(0, T), \\
p(T)=0 \\
p^{\prime}(T)=0 .
\end{array}\right.
$$

Proposition 4. Equation (104) admits a unique weak solution $p \in \mathcal{S}(0, T)$.
Proof. By the reversion in time $t \longrightarrow T-t$ and the reversion $\sigma \longrightarrow T-\sigma$ in equation (104), we can obtain the following:

$$
\left\{\begin{array}{l}
p^{\prime \prime}(T-t)+A p(T-t)+\int_{T-t}^{T} k(0) A p \mathrm{~d} s-\int_{T-t}^{T} \int_{T-s}^{0} k^{\prime}(T-\sigma-s) A p(T-\sigma) \mathrm{d} \sigma \mathrm{d} s \\
-(\gamma+1) \int_{T-t}^{T}\left|y\left(q^{*}\right)\right|^{\gamma} p^{\prime} \mathrm{d} s+\int_{T-t}^{T} q^{*} p^{\prime} \mathrm{d} s=g(T-t), \quad \text { in }(0, T), \\
p(T-0)=0, \\
p^{\prime}(T-0)=0,
\end{array}\right.
$$

where $g(\cdot)=y^{\prime}\left(q^{*} ; \cdot\right)-y_{d}(\cdot)$. By changing $s \longrightarrow T-s$ in equation (105) and then changing every function in the reversed equation like this $p(T-\cdot) \longrightarrow p(\cdot)$, we can have the following initial value problem:

$$
\left\{\begin{array}{l}
p^{\prime \prime}+A p+\int_{0}^{t} k(0) A p \mathrm{~d} s+\int_{0}^{t} k^{\prime} * A p \mathrm{~d} s+(\gamma+1) \int_{0}^{t}\left|y\left(q^{*}\right)\right|^{\gamma} p^{\prime} \mathrm{d} s-\int_{0}^{t} q^{*} p^{\prime} \mathrm{d} s=g, \quad \text { in }(0, T) \\
p(0)=0 \\
p^{\prime}(0)=0
\end{array}\right.
$$


Now, we apply the well-known Faedo-Galerkin procedure to equation (106): since $V$ is separable, there exists a basis $\left\{w_{m}\right\}_{m=1}^{\infty}$ in $V$ such that $\left\{w_{m}\right\}_{m=1}^{\infty}$ is a complete orthonormal system in $H$ and free and total in $V$. For each $m \in N$, we define an approximate solution of equation (106) by

$$
p_{m}(t)=\sum_{j=1}^{m} g_{j m}(t) w_{j},
$$

where $p_{m}(t)$ satisfies

$$
\left\{\begin{array}{l}
\left(p_{m}^{\prime \prime}(t), w_{j}\right)_{2}+\left(\left(p_{m}(t), w_{j}\right)\right)_{V}+\int_{0}^{t}\left(\left(k(0) p_{m}+k^{\prime} * p_{m}, w_{j}\right)\right)_{V} \mathrm{~d} s \\
+\int_{0}^{t}\left((\gamma+1)\left|y\left(q^{*}\right)\right|^{\gamma} p_{m}^{\prime}-q^{*} p_{m}^{\prime}, w_{j}\right)_{2} \mathrm{~d} s=\left(g, w_{j}\right)_{2}, \quad \text { in }(0, T), 1 \leq j \leq m, \\
p_{m}(0)=p_{m}^{\prime}(0)=0 .
\end{array}\right.
$$

We multiply both sides of equation (108) by $g_{j m}{ }^{\prime}(t)$ and sum over $j$ to obtain

$$
\begin{aligned}
& \frac{1}{2} \frac{\mathrm{d}}{\mathrm{d} t}\left(\left\|p_{m}^{\prime}(t)\right\|_{2}^{2}+\left\|p_{m}(t)\right\|_{V}^{2}\right)+\int_{0}^{t}\left(\left(k(0) p_{m}+k^{\prime} * p_{m}, p_{m}^{\prime}(t)\right)\right)_{V} \mathrm{~d} s \\
& \quad+\int_{0}^{t}\left((\gamma+1)\left|y\left(q^{*}\right)\right|^{\gamma} p_{m}^{\prime}-q^{*} p_{m}^{\prime}, p_{m}^{\prime}(t)\right)_{2} \mathrm{~d} s \\
& =\left(g, p_{m}^{\prime}(t)\right)_{2} .
\end{aligned}
$$

Integrating (109) from 0 to $t$ and by integration by parts, we obtain

$$
\begin{aligned}
& \frac{1}{2}\left(\left\|p_{m}^{\prime}(t)\right\|_{2}^{2}+\left\|p_{m}(t)\right\|_{V}^{2}\right)+\int_{0}^{t}\left(\left(k(0) p_{m}+k^{\prime} * p_{m}, p_{m}(t)\right)\right)_{V} \mathrm{~d} s \\
& \quad-\int_{0}^{t}\left(\left(k(0) p_{m}+k^{\prime} * p_{m}, p_{m}\right)\right)_{V} \mathrm{~d} s \\
& \quad+\int_{0}^{t}\left((\gamma+1)\left|y\left(q^{*}\right)\right|^{\gamma} p_{m}^{\prime}-q^{*} p_{m}^{\prime}, p_{m}(t)\right)_{2} \mathrm{~d} s \\
& \quad-\int_{0}^{t}\left((\gamma+1)\left|y\left(q^{*}\right)\right|^{\gamma} p_{m}^{\prime}-q^{*} p_{m}^{\prime}, p_{m}\right)_{2} \mathrm{~d} s \\
& =\int_{0}^{t}\left(g, p_{m}^{\prime}\right)_{2} \mathrm{~d} s .
\end{aligned}
$$

By (59), we can obtain the following estimations:

$$
\begin{aligned}
& \left|\int_{0}^{t}\left((\gamma+1)\left|y\left(q^{*}\right)\right|^{\gamma} p_{m}^{\prime}, p_{m}(t)\right)_{2} \mathrm{~d} s\right| \\
& \leq C \int_{0}^{t}\left\|p_{m}^{\prime}\right\|_{2}\left\|p_{m}(t)\right\|_{V} \mathrm{~d} s \\
& \leq \varepsilon\left\|p_{m}(t)\right\|_{V}^{2}+\frac{C}{\varepsilon} \int_{0}^{t}\left\|p_{m}^{\prime}\right\|_{2}^{2} \mathrm{~d} s, \\
& \left|\int_{0}^{t}\left((\gamma+1)\left|y\left(q^{*}\right)\right|^{\gamma} p_{m}^{\prime}, p_{m}\right)_{2} \mathrm{~d} s\right| \\
& \leq C \int_{0}^{t}\left\|p_{m}^{\prime}\right\|_{2}\left\|p_{m}(t)\right\|_{V} \mathrm{~d} s \\
& \leq C \int_{0}^{t}\left(\left\|p_{m}^{\prime}\right\|_{2}^{2}+\left\|p_{m}(t)\right\|_{V}^{2}\right) \mathrm{d} s .
\end{aligned}
$$

And we also have the following estimations:

$$
\begin{aligned}
&\left|\int_{0}^{t}\left(q^{*} p_{m}^{\prime}, p_{m}(t)\right)_{2} \mathrm{~d} s\right| \leq\left\|p_{m}(t)\right\|_{2} \int_{0}^{t}\left|q^{*}\right|\left\|p_{m}^{\prime}\right\|_{2} \mathrm{~d} s \\
& \leq C\left\|p_{m}(t)\right\|_{V} \int_{0}^{t}\left|q^{*}\right|\left\|p_{m}^{\prime}\right\|_{2} \mathrm{~d} s \\
& \leq \varepsilon\left\|p_{m}(t)\right\|_{V}^{2}+\frac{C}{\varepsilon} \int_{0}^{t}\left|q^{*}\right|^{2}\left\|p_{m}^{\prime}\right\|_{2}^{2} \mathrm{~d} s \\
&\left|\int_{0}^{t}\left(q^{*} p_{m}^{\prime}, p_{m}\right)_{2} \mathrm{~d} s\right| \leq \int_{0}^{t}\left|q^{*}\right|\left\|p_{m}^{\prime}\right\|_{2}\left\|p_{m}\right\|_{2} \mathrm{~d} s \\
& \leq C \int_{0}^{t}\left|q^{*}\right|\left\|p_{m}^{\prime}\right\|_{2}\left\|p_{m}\right\|_{V} \mathrm{~d} s \\
& \leq C \int_{0}^{t}\left(\left|q^{*}\right|^{2}\left\|p_{m}^{\prime}\right\|_{2}^{2}+\left\|p_{m}\right\|_{V}^{2}\right) \mathrm{d} s
\end{aligned}
$$

By (111)-(114) with a properly determined $\varepsilon$ and estimating other terms in (110) as in the proof of Theorem 1, we can arrive at

$$
\begin{aligned}
& \left\|p_{m}^{\prime}(t)\right\|_{2}^{2}+\left\|p_{m}(t)\right\|_{V}^{2} \\
& \leq C\|g\|_{L^{2}(0, T ; H)}^{2}+C \int_{0}^{t}\left(1+\left|q^{*}\right|^{2}\right)\left(\left\|p_{m}^{\prime}\right\|_{2}^{2}+\left\|p_{m}\right\|_{V}^{2}\right) \mathrm{d} s .
\end{aligned}
$$

Thus, by Gronwall's lemma, we see that

$$
p_{m} \in \mathscr{W}(0, T) \cap L^{\infty}(0, T ; V) \cap W^{1, \infty}(0, T ; H) .
$$


Then, following similar arguments in [18], we can ensure that equation (104) admits a weak solution $p \in \mathcal{S}(0, T)$. Uniqueness will be proved straightforwardly for $g=0$.

This completes the proof.

Now, we investigate the first-order optimality conditions for minimax optimal control problem (54) for quadratic cost function (103).

Theorem 5. If $\alpha$ and $\beta$ in cost (103) are large enough and the exponent $\gamma \geq 1$, then an optimal control $c^{*} \in \mathcal{U}_{\text {ad }}$ and $a$ disturbance $\eta^{*} \in \mathscr{V}_{\text {ad }}$, namely, an optimal pair $q^{*}=\left(c^{*}, \eta^{*}\right) \in \mathscr{F}_{\text {ad }}$ satisfying (54), can be given by

$$
\begin{gathered}
c^{*}=\max \left\{c_{l}, \min \left\{\frac{\left(y\left(q^{*}\right), p^{\prime}\right)_{2}}{\alpha}, c_{u}\right\}\right\}, \\
\eta^{*}=\max \left\{\eta_{l}, \min \left\{-\frac{\left(y\left(q^{*}\right), p^{\prime}\right)_{2}}{\beta}, \eta_{u}\right\}\right\},
\end{gathered}
$$

where $p$ is the weak solution of equation (104).

Remark 3. To get the optimality condition in Theorem 5, we need to multiply the weak form of equation (104) by $z^{\prime}$. Since we just know that $z^{\prime} \in C([0, T] ; H)$, it would be a just formal procedure. To overcome this difficulty, we employ the regularization method of Lions ([14], pp. 286-288) which was used to deal with linear hyperbolic problems (cf. [15]).

Proof of Theorem 5. By the assumptions of Theorem 5, we can verify through Theorem 3 that there exists an optimal pair in (54) with cost (103). Let $\left(c^{*}, \eta^{*}\right) \in \mathscr{F}_{\text {ad }}$ be an optimal pair in (54) with cost (103) and $y\left(q^{*}\right)$ be the corresponding weak solution of equation (52).

By similar arguments in Theorem 4, we can know that cost (103) is also (strongly) Gâteaux differentiable at $\left(c^{*}, \eta^{*}\right)$ in the direction $w=(h, l) \in\left[L^{2}(0, T)\right]^{2}$ which satisfies for sufficiently small $\epsilon>0$ that $\left(c^{*}, \eta^{*}\right)+\varepsilon w \in \mathscr{F}_{\text {ad }}$. Thus, we deduce the following:

$$
\begin{aligned}
& D \mathscr{J}\left(c^{*}, \eta^{*}\right)(h, l)=\lim _{\varepsilon \longrightarrow 0^{+}} \frac{\mathscr{J}\left(c^{*}+\varepsilon h, \eta^{*}+\varepsilon l\right)-\mathscr{J}\left(c^{*}, \eta^{*}\right)}{\varepsilon} \\
= & \int_{0}^{T}\left(y^{\prime}\left(q^{*}\right)-y_{d}, z^{\prime}\right)_{2} \mathrm{~d} t+\alpha\left(c^{*}, h\right)_{L^{2}(0, T)}-\beta\left(\eta^{*}, l\right)_{L^{2}(0, T)},
\end{aligned}
$$

where $z=D y\left(q^{*}\right) w$ is a solution of equation (71).

As explained above, we prove this theorem by the regularization method. For this purpose, we extend the time domains of equations (71) and (104) to $\mathbf{R}_{t}$ by introducing $\psi$ and $\theta$ as the solutions of

$$
\begin{aligned}
& \begin{cases}\psi^{\prime \prime}+A \psi+k * A \psi+(\gamma+1)\left|y\left(q^{*}\right)\right|^{\gamma} \psi= \begin{cases}q^{*} \psi+(h+l) y\left(q^{*}\right), & \text { in }(0, T), \\
0, & \text { otherwise },\end{cases} \\
\psi(0)=0, \\
\psi^{\prime}(0)=0,\end{cases} \\
& \begin{cases}\theta^{\prime \prime}+A \theta+\int_{t}^{T}\left(\mathscr{K}(s, \theta)+\mathscr{G}\left(q^{*}, \theta^{\prime}\right)\right) \mathrm{d} s= \begin{cases}y^{\prime}\left(q^{*}\right)-y_{d}, & \text { in }(0, T), \\
0, & \text { otherwise }\end{cases} \\
\theta(T)=0, \\
\theta^{\prime}(T)=0,\end{cases}
\end{aligned}
$$

where

In fact, we have

$$
\begin{aligned}
& \mathscr{K}(s, \theta)=k(0) A \theta+\int_{s}^{T} k^{\prime}(\sigma-s) A \theta \mathrm{d} \sigma, \\
& \mathscr{G}\left(q^{*}, \theta^{\prime}\right)=-(\gamma+1)\left|y\left(q^{*}\right)\right|^{\gamma} \theta^{\prime}+q^{*} \theta^{\prime} .
\end{aligned}
$$

$$
\begin{aligned}
\psi & =z, \quad \text { in }(0, T), \\
\theta & =p, \quad \text { in }(0, T), \\
\int_{0}^{T}\left(y^{\prime}\left(q^{*}\right)-y_{d}, z^{\prime}\right)_{2} \mathrm{~d} t & =\int_{\mathbf{R}_{t}}\left(\theta^{\prime \prime}+A \theta+\int_{t}^{T}\left(\mathscr{K}(s, \theta)+\mathscr{G}\left(q^{*}, \theta^{\prime}\right)\right) \mathrm{d} s, \psi^{\prime}\right)_{2} \mathrm{~d} t .
\end{aligned}
$$


For the simplicity, we shall denote the scalar product in $H$ or the antiduality $V, V^{\prime}$ by $[\cdot, \cdot]$. Let $\rho_{n}$ be a regularizing sequence on $\mathbf{R}_{t}$. Then, the right-hand side of (124) becomes

$$
\lim _{n \longrightarrow \infty} \int_{\mathbf{R}_{t}}\left[\left(\theta^{\prime \prime}+A \theta+\int_{t}^{T}\left(\mathscr{K}(s, \theta)+\mathscr{G}\left(q^{*}, \theta^{\prime}\right)\right) \mathrm{d} s\right) * \rho_{n}, \psi^{\prime} * \rho_{n}\right] \mathrm{d} t .
$$

Now, we integrate by parts of (125). Then, we put

$$
\begin{aligned}
X_{n}=\int_{\mathbf{R}_{t}}\left(\left[-\theta^{\prime} * \rho_{n}, \psi^{\prime \prime *} \rho_{n}\right]+\left[(A \theta)^{*} \rho_{n}, \psi^{\prime} * \rho_{n}\right]\right. \\
\left.+\left[\left(\mathscr{K}(\cdot, \theta)+\mathscr{G}\left(q^{*}, \theta^{\prime}\right)\right) * \rho_{n}, \psi^{*} \rho_{n}\right]\right) \mathrm{d} t .
\end{aligned}
$$

Using equation (119), that is, $\psi^{\prime \prime}=-A \psi-k * A \psi+\mathscr{G}$ $\left(q^{*}, \psi\right)+(h+l) y\left(q^{*}\right),(126)$ can be given again by

$$
X_{n}=\int_{\mathbf{R}_{t}}\left[-\theta^{\prime} * \rho_{n},\left((h+l) y\left(q^{*}\right)\right) * \rho_{n}\right] \mathrm{d} t+\sum_{i=1}^{3} Y_{n}^{i},
$$

where

$$
\begin{aligned}
Y_{n}^{1} & =\int_{\mathbf{R}_{t}}\left(\left[\theta^{\prime} * \rho_{n},(A \psi) * \rho_{n}\right]+\left[(A \theta) * \rho_{n}, \psi^{\prime} * \rho_{n}\right]\right) \mathrm{d} t, \\
Y_{n}^{2} & =\int_{\mathbf{R}_{t}}\left(\left[\theta^{\prime} * \rho_{n},(k * A \psi) * \rho_{n}\right]+\left[\mathscr{K}(\cdot, \theta) * \rho_{n}, \psi * \rho_{n}\right]\right) \mathrm{d} t, \\
Y_{n}^{3} & =\int_{\mathbf{R}_{t}}\left(\left[-\theta^{\prime} * \rho_{n}, \mathscr{G}\left(q^{*}, \psi\right) * \rho_{n}\right]+\left[\mathscr{G}\left(q^{*}, \theta^{\prime}\right) * \rho_{n}, \psi * \rho_{n}\right]\right) \mathrm{d} t .
\end{aligned}
$$

We immediately know $Y_{n}^{1}=0$ by integration by parts. By integration by parts, we can see that

$$
\begin{aligned}
Y_{n}^{2}= & \int_{\mathbf{R}_{t}}\left(-\left[\theta * \rho_{n},\left(k(0) A \psi+k^{\prime} * A \psi\right) * \rho_{n}\right]\right. \\
& \left.+\left[\mathscr{K}(\cdot, \theta) * \rho_{n}, \psi * \rho_{n}\right]\right) \mathrm{d} t .
\end{aligned}
$$

Thus,

$$
Y_{n}^{2} \longrightarrow \int_{\mathbf{R}_{t}}\left(-\left[\theta, k(0) A \psi+k^{\prime} * A \psi\right]+[\mathscr{K}(t, \theta), \psi]\right) \mathrm{d} t,
$$

as $n \longrightarrow \infty$. From (121) and Fubini's theorem, we know that the right-hand side of (130) is 0 . Therefore, we can obtain $Y_{n}^{2} \longrightarrow 0$ as $n \longrightarrow \infty$. And we can easily verify that $Y_{n}^{3} \longrightarrow 0$ as $n \longrightarrow \infty$. Consequently, we can obtain

$$
\begin{aligned}
\lim _{n \longrightarrow \infty} X_{n} & =\int_{\mathbf{R}_{t}}\left[-\theta^{\prime},(h+l) y\left(q^{*}\right)\right] \mathrm{d} t \\
& =\int_{0}^{T}\left(-p^{\prime},(h+l) y\left(q^{*}\right)\right)_{2} \mathrm{~d} t .
\end{aligned}
$$

Since $\left(c^{*}, \eta^{*}\right) \in \mathscr{F}_{\text {ad }}$ is an optimal pair in (54), we know

$$
\begin{aligned}
& D_{c} J\left(c^{*}, \eta^{*}\right)(h) \geq 0, \\
& D_{\eta} J\left(c^{*}, \eta^{*}\right)(l) \leq 0, \quad(h, l) \in\left[L^{2}(0, T)\right]^{2} .
\end{aligned}
$$

From (118), (124), and (131), we know that (132) implies

$$
\begin{gathered}
\left(\alpha c^{*}-\left(y\left(q^{*}\right), p^{\prime}\right)_{2}, h\right)_{L^{2}(0, T)} \geq 0, \\
\left(-\beta \eta^{*}-\left(y\left(q^{*}\right), p^{\prime}\right)_{2}, l\right)_{L^{2}(0, T)} \leq 0,
\end{gathered}
$$

where $(h, l) \in\left[L^{2}(0, T)\right]^{2}$. By similar arguments in the proof of Theorem 4, we can deduce from (133) that

$$
\begin{aligned}
& c^{*}=\max \left\{c_{l}, \min \left\{\frac{\left(y\left(q^{*}\right), p^{\prime}\right)_{2}}{\alpha}, c_{u}\right\}\right\}, \\
& \eta^{*}=\max \left\{\eta_{l}, \min \left\{-\frac{\left(y\left(q^{*}\right), p^{\prime}\right)_{2}}{\beta}, \eta_{u}\right\}\right\} .
\end{aligned}
$$

This completes the proof.

\section{Conclusion}

In this paper, we study the bilinear minimax optimal control problems for a semilinear viscoelastic equation with long memory. A global well-posedness theorem regarding the solutions to its Cauchy problem is given. We show the Fréchet differentiability of the nonlinear solution map from the bilinear control input to the solution of the state equation given by the above equation. We formulate the minimax optimal control problem for the state equation. By using and analyzing the properties of the Fréchet derivative of the nonlinear solution map, we show the existence of optimal pairs and find their necessary optimality conditions corresponding to the practical observation cases.

\section{Data Availability}

All the data sets generated for this study are included within this manuscript.

\section{Conflicts of Interest}

The author declares no conflicts of interest.

\section{Authors' Contributions}

The author contributed solely to the writing of this paper and read and approved the manuscript.

\section{Acknowledgments}

This work was supported by the National Research Foundation of Korea (NRF) grant funded by the Korea Government (MSIT) (NRF-2020R1F1A1A01074403).

\section{References}

[1] M. M. Cavalcanti, V. N. Domingos Cavalcanti, and J. A. Soriano, "Exponential decay for the solution of semilinear viscoelastic wave equations with localized damping, Electron," Journal Differential Equations, vol. 44, pp. 1-14, 2002.

[2] M. M. Cavalcanti, V. N. Domingos Cavalcanti, and J. A. Soriano, "Global existence and asymptotic stability for viscoelastic problems," Differential Integral Equations, vol. 15, pp. 731-748, 2002. 
[3] T.-J. Xiao and J. Liang, "Coupled second order semilinear evolution equations indirectly damped via memory effects," Journal of Differential Equations, vol. 254, no. 5, pp. 21282157, 2013.

[4] S. A. Messaoudi, "Blow-up of positive-initial-energy solutions of a nonlinear viscoelastic hyperbolic equation," Journal of Mathematical Analysis and Applications, vol. 320, no. 2, pp. 902-915, 2006.

[5] M. E. Bradley and S. Lenhart, "Bilinear optimal control of a Kirchhoff plate," Systems \& Control Letters, vol. 22, no. 1, pp. 27-38, 1994.

[6] M. E. Bradley and S. Lenhart, "Bilinear spatial control of the velocity term in a Kirchhoff plate equation," Electron Journal Differential Equations, vol. 27, pp. 1-15, 2001.

[7] M. E. Bradley, S. Lenhart, and J. Yong, "Bilinear optimal control of the velocity term in a Kirchhoff plate equation," Journal of Mathematical Analysis and Applications, vol. 238, no. 2, pp. 451-467, 1999.

[8] A. Belmiloudi, "Bilinear minimax control problems for a class of parabolic systems with applications to control of nuclear reactors," Journal of Mathematical Analysis and Applications, vol. 327, no. 1, pp. 620-642, 2007.

[9] J.-s. Hwang, "Fréchet differentiability for an extensible beam equation and its application to bilinear minimax control problems," Journal of Mathematical Analysis and Applications, vol. 474, no. 1, pp. 157-178, 2019.

[10] N. Arada and J.-P. Raymond, "Minimax control of parabolic systems with state constraints," SIAM Journal on Control and Optimization, vol. 38, no. 1, pp. 254-271, 1999.

[11] I. Lasiecka and R. Triggiani, Control Theory for Partial Differential Equations: Continuous and Approximation Theories, I, Cambridge University Press, Cambridge, UK, 2000.

[12] X. Li and J. Yong, Optimal Control Theory for Infinite Dimensional Systems, Birkhäuser, Boston, MA, USA, 1995.

[13] V. Barbu and T. Precupanu, Convexity and Optimization in Banach Spaces, Reidel, Dordrecht, Germany, 1986.

[14] J. L. Lions, Optimal Control of Systems Governed by Partial Differential Equations, Springer-Verlag Berlin Heidelberg, Berlin, Germany, 1971.

[15] J.-s. Hwang, "Optimal control problems for an extensible beam equation," Journal of Mathematical Analysis and Applications, vol. 353, no. 1, pp. 436-448, 2009.

[16] R. A. Adams, Sobolev Spaces, Academic Press, Cambridge, MA, USA, 1975.

[17] R. Teman, "Infinite-dimensional dynamical systems in mechanics and physics," Applied Mathematics Science, vol. 68, 1997.

[18] J. Hwang and S. Nakagiri, "On semi-linear second order Volterra integro-differential equations in Hilbert space," Taiwanese Journal of Mathematics, vol. 12, pp. 679-701, 2008.

[19] J. L. Lions and E. Magenes, Non-Homogeneous Boundary Value Problems and Applications I, II, Springer-Verlag Berlin Heidelberg, Berlin, Germany, 1972.

[20] R. Dautray and J. L. Lions, "Mathematical analysis and numerical methods for science and technology," Evolution Problems I, Springer-Verlag, Berlin, Germany, 1992.

[21] J. Simon, "Compact sets in the space $L^{p}(0, T ; B)$, , Annali di Matematica Pura ed Applicata, vol. 146, no. 4, pp. 65-96, 1987. 